\title{
Anatomy of Einstein manifolds
}

\author{
Jongmin Park, Jaewon Shin, ${ }^{\dagger}$ and Hyun Seok Yang $\oplus^{\ddagger}$ \\ Department of Physics and Photon Science, Gwangju Institute of Science and Technology, \\ Gwangju 61005, Korea
}

(Received 15 September 2021; accepted 24 February 2022; published 8 March 2022)

\begin{abstract}
An Einstein manifold in four dimensions has some configuration of $S U(2)_{+}$Yang-Mills instantons and $S U(2)_{-}$anti-instantons associated with it. This fact is based on the fundamental theorems that the four-dimensional Lorentz group $\operatorname{Spin}(4)$ is a direct product of two groups $S U(2)_{ \pm}$and the vector space of 2-forms decomposes into the space of self-dual and anti-self-dual 2-forms. It explains why the four-dimensional spacetime is special for the stability of Einstein manifolds. We now consider whether such a stability of four-dimensional Einstein manifolds can be lifted to a fivedimensional Einstein manifold. The higher-dimensional embedding of four-manifolds from the viewpoint of gauge theory is similar to the grand unification of the Standard Model, since the group $S O(4) \cong \operatorname{Spin}(4) / \mathbb{Z}_{2}=S U(2)_{+} \otimes S U(2)_{-} / \mathbb{Z}_{2}$ must be embedded into the simple group $S O(5)=S p(2) / \mathbb{Z}_{2}$. Our group-theoretic approach reveals the anatomy of Riemannian manifolds quite similar to the quark model of hadrons in which two independent Yang-Mills instantons represent a substructure of Einstein manifolds.
\end{abstract}

DOI: 10.1103/PhysRevD.105.064015

\section{INTRODUCTION}

The hadrons we know all fall into multiplets that reflect underlying internal symmetries. To express this fact in a simple and concrete way, it was hypothesized that hadrons are composed of more elementary constituents with basic symmetries, called quarks. The $S U(3)$ multiplet structure of the hadrons (baryons and mesons) strongly hinted at the existence of a substructure [1,2]. According to the quark model [3], all hadrons are made up of quarks and antiquarks, bound together in different ways. Even in the absence of knowledge about the potential which binds quarks and antiquarks, the model was very predictive. The triple tensor product of the fundamental representation 3 of the $S U(3)$ flavor symmetry leads to octets and a decuplet of baryons, $3 \otimes \mathbf{3} \otimes \mathbf{3}=\mathbf{1} \oplus \mathbf{8} \oplus \mathbf{8} \oplus \mathbf{1 0}$, in addition to a singlet. This classification works also for mesons: $\mathbf{3} \otimes \overline{\mathbf{3}}=\mathbf{1} \oplus \mathbf{8}$. This quark model eventually led to the introduction of color degrees of freedom and the construction of quantum chromodynamics $[4,5]$.

\footnotetext{
*whdalsd1776@gist.ac.kr

†1thegreat@gm.gist.ac.kr

"hsyang@gist.ac.kr
}

Published by the American Physical Society under the terms of the Creative Commons Attribution 4.0 International license. Further distribution of this work must maintain attribution to the author(s) and the published article's title, journal citation, and DOI. Funded by SCOAP ${ }^{3}$.
A special feature, which permeates four-dimensional geometry, is the fact that $\operatorname{Spin}(4)$ splits into a product of two groups:

$$
\operatorname{Spin}(4)=S U(2)_{+} \times S U(2)_{-} .
$$

The group Spin(4) is a double cover of the fourdimensional Euclidean Lorentz group $S O(4)$, i.e., $S O(4) \cong S U(2)_{+} \times S U(2)_{-} / \mathbb{Z}_{2}$. The splitting of $\operatorname{Spin}(4)$ is isomorphically related to the decomposition of the 2 -forms on a four-manifold. Using the Hodge $*$ operator acting on exterior 2-forms, one can split 2-forms into selfdual and anti-self-dual 2-forms. The splitting can be applied to the curvature form of any bundle with connection over an oriented four-manifold. The canonical splitting of the vector spaces leads to the irreducible decomposition of Riemann curvature tensor $R \in C^{\infty}\left(\mathfrak{g} \otimes \Omega^{2}\right)$ as [6]

$$
R=R_{(++)} \oplus R_{(+-)} \oplus R_{(-+)} \oplus R_{(--)},
$$

where the subscript $( \pm \pm)$ refers to the splitting of the vector spaces $\mathfrak{g} \equiv \operatorname{so}(4)=s u(2)_{+} \oplus s u(2)_{-}$and $\Omega^{2} \equiv$ $\Lambda^{2} T^{*} M=\Omega_{+}^{2} \oplus \Omega_{-}^{2}$. This splitting of the vector spaces occupies a central position for the Donaldson theory of four-manifolds and has been well known in mathematical literatures (see, for example, Chaps. 1.G and 1.H in Ref. [7] and Secs. 1.1 and 2.1 in Ref. [8]).

Imposing the Einstein equations, $R_{\mu \nu}=\lambda g_{\mu \nu}$, leads to the condition $R_{(+-)}=R_{(-+)}^{T}=0$ (6.32 in Ref. [7] and lemma 
in Ref. [9]). In this case, the Riemann curvature tensor satisfies the self-duality equations $* R_{( \pm \pm)}=R_{( \pm \pm)} *=$ $\pm R_{( \pm \pm)}$, where the Hodge $*$ operator $* R$ acts on the first two indices $[a b]$ of the curvature tensor $R_{a b c d}$ and $R *$ acts on the last two indices $[c d]$. Therefore, an Einstein manifold consists of $S U(2)_{+}$instantons and $S U(2)_{-}$anti-instantons defined over itself $[9,10]$. The instantons in the $S U(2)_{+}$ group live in a different representation space from the anti-

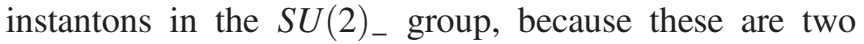
independent factors for the product group (1.1). This means that $R_{(++)}$and $R_{(--)}$correspond to two independent components defined by self-dual and anti-self-dual spin connections, respectively, acting on the chiral and antichiral spin bundles. This special feature of four-dimensional gravity has been originated from the splitting of the product group (1.1).

A four-dimensional Einstein manifold has the irreducible decomposition defined by the curvature tensor $R=$ $R_{(++)} \oplus R_{(--)}$which brings about two independent gravitational components. However, such division into two independent instanton sectors explains the stability of Einstein manifolds. It turns out [11] that the topological invariants carried by an Einstein manifold are determined by the configuration of $S U(2)_{+}$instantons and $S U(2)_{-}$ anti-instantons, as will be reviewed in Sec. II. Therefore, an Einstein manifold has a substructure like hadrons. An interesting physics arises if the four-dimensional gravity is regarded as being obtained from a five-dimensional gravity through the Kaluza-Klein compactification [12]. The Riemann curvature tensor in five dimensions takes values in the Lie algebra of the Lorentz group $S O(5)$. The group $S O(5)$ is a simple group unlike the four-dimensional Lorentz group $S O(4)=S U(2)_{+} \times S U(2)_{-} / \mathbb{Z}_{2}$. Since the group $S O(4)$ must be embedded into the simple group $S O(5)=S p(2) / \mathbb{Z}_{2}$ in the five-dimensional gravity, we expect that two independent components caused by the separation of Riemann curvature tensors will be combined into a single gravitation force in five dimensions. Moreover, the electromagnetism and a scalar field obtained from a five-dimensional metric through the KaluzaKlein reduction should also appear in the same multiplet in an irreducible representation (irrep) of the Lorentz group $S O(5)$.

This unification scheme is similar to the grand unification of the Standard Model, since the group $S O(4) \cong$ $S U(2)_{+} \otimes S U(2)_{-} / \mathbb{Z}_{2}$ must be embedded into the simple group $S O(5)=S p(2) / \mathbb{Z}_{2}$ although the Kaluza-Klein theory is reduced from a five-dimensional gravity. The Standard Model has a product gauge group $S U(3) \times$ $S U(2) \times U(1)$ to describe the electroweak and strong forces. In the grand unified theory (GUT), the product gauge group in the Standard Model is embedded into a single gauge group, for example, $S U(5)$ or $S O(10)$ (see, e.g., Chaps. 18 and 24 in Ref. [13]). The leptons and quarks in the GUT appear in the same multiplet in a larger symmetry. The unification of forces with a larger simple group typically opens a new decay channel of protons into leptons and so introduces a novel instability of a stable particle in the Standard Model. We will see how two instanton sectors of four-dimensional Einstein manifolds are similarly combined into a five-dimensional Einstein manifold. The embedding of $S U(2)_{+}$instantons and $S U(2)_{-}$anti-instantons into a five-dimensional Einstein manifold may similarly develop a novel instability like the proton decay in the GUT. A speculative reason for this assumption is that there is no natural topological invariant such as the Euler characteristic or the Hirzebruch signature in five dimensions $[7,14]$ that guarantees the stability of five-dimensional Einstein manifolds.

The motivation for the present work lies in providing a fresh point of view for the topological structure of Einstein manifolds using the group properties of $S O(4) \cong$ $S U(2)_{+} \otimes S U(2)_{-} / \mathbb{Z}_{2}$ and $S O(5)=S p(2) / \mathbb{Z}_{2}$. We hope it will provide a deeper insight into the nature of the stability of Riemannian manifolds. We will mostly refer to the perturbative stability of Einstein manifolds regarding the second variation of the Einstein-Hilbert action with a fixed volume at a background Einstein metric (see Chaps. 4 and 12 in Ref. [7]). But a nonperturbative instability may be induced by instanton transitions. We will not try to exhaust all the details but initiate a work along this direction.

This paper is organized as follows. In Sec. II, we briefly review how the decomposition of Riemann curvature tensor (1.2) is derived from the splitting of the vector spaces $\mathfrak{g} \equiv$ $s o(4)=s u(2)_{+} \oplus s u(2)_{-}$and $\Omega^{2} \equiv \Lambda^{2} T^{*} M=\Omega_{+}^{2} \oplus \Omega_{-}^{2}$. We also discuss how topological invariants of Einstein manifolds such as the Euler characteristic and the Hirzebruch signature are determined by the configuration of $S U(2)_{+}$instantons and $S U(2)_{-}$anti-instantons to illuminate a substructure of Einstein manifolds. In Sec. III, we consider a five-dimensional Einstein manifold and its Kaluza-Klein reduction. We expand the five-dimensional Riemann curvature tensor in the basis of $\operatorname{sp}(2) \cong$ so(5) Lie algebra which generalizes the decomposition (1.2) to five dimensions. After the Kaluza-Klein reduction, the Lorentz symmetry $S O(5)$ is spontaneously broken to $S O(4) \times U(1)$, where $U(1)$ is originated from the isometries of the Kaluza-Klein circle [12]. According to the symmetry breaking pattern, we further decompose the fivedimensional Riemann curvature tensor in the basis of $s o(4) \cong s u(2)_{+} \oplus s u(2)_{-}$Lie algebra. This decomposition is useful to see how $U(1)$ gauge fields and a scalar field deform the instanton structure of four-dimensional Einstein manifolds and to understand how these deformed geometries are nicely combined into a five-dimensional Einstein manifold. In Sec. IV, we consider particular cases to consolidate that all these deformations can be organized into a single five-dimensional Einstein manifold once the fifth dimension is opened so that the Lorentz symmetry is enhanced to $S O(5)$. In Sec. V, we discuss some important 
issues and generalization to noncompact Einstein manifolds that we have not addressed in this paper and speculate a possible origin of novel instabilities of Einstein manifolds in five dimensions.

In the Appendix A, we provide some details about the Lie algebras $s o(4) \cong s u(2)_{+} \oplus s u(2)_{-}$and $s p(2) \cong s o(5)$. The geometric details of five-dimensional gravity and Kaluza-Klein gravity, especially in the vielbein formalism, appear in Appendix B. Appendix C contains the group structure analysis of Riemann curvature tensors and the decomposition of Ricci tensors and Ricci scalar in the so(4) Lie algebra basis.

\section{EINSTEIN MANIFOLDS AS YANG-MILLS INSTANTONS}

It is known $[15,16]$ that Einstein manifolds are stable, at least perturbatively. It is a bit mysterious, recalling that gravity is also described within the framework of field theory. One way to understand the stability is to notice that an Einstein manifold carries nontrivial topological invariants such as the Euler characteristic $\chi$ and Hirzebruch signature $\tau$ [14]. The gauge theory formulation of gravity reveals a beautiful aspect of the stability. It turns out $[9,10]$ that an Einstein manifold in four dimensions has a configuration of $S U(2)_{+}$Yang-Mills instantons and $S U(2)_{-}$ anti-instantons. Two kinds of instantons are independent of each other, because they belong to different gauge groups. ${ }^{1}$ Furthermore, instantons can be superposed to make multiinstantons. In principle, it is possible to have a tower of Einstein manifolds by superposing $S U(2)$ instantons in each gauge group. The multi-Taub-NUT spaces [17] could serve as an example of such a tower (with only one type of instanton used). Of course, a compact manifold has subtle global obstructions for gluing multi-instantons (see Chap. 7 in Ref. [8]). Let us briefly recapitulate this aspect of the stability.

Consider an Einstein manifold $(M, g)$. The metric on $M$ takes the form

$$
d s_{4}^{2}=g_{\mu \nu}(x) d x^{\mu} d x^{\nu}=e^{a} \otimes e^{a} .
$$

Using the metric, one can determine the spin connections $\omega^{a}{ }_{b}=\omega_{b \mu}^{a} d x^{\mu}$ and curvature tensors $R_{b}^{a}=\frac{1}{2} R_{b \mu \nu}^{a} d x^{\mu} \wedge$ $d x^{\nu}$ by solving the structure equations $[14,18]$

$$
\begin{aligned}
& T^{a}=d e^{a}+\omega_{b}^{a} \wedge e^{b}=0, \\
& R^{a}{ }_{b}=d \omega^{a}{ }_{b}+\omega^{a}{ }_{c} \wedge \omega^{c}{ }_{b} .
\end{aligned}
$$

\footnotetext{
${ }^{1}$ This reasoning may not be complete, because a new instability may be developed through the interaction between instantons. Moreover, $\mathbb{T}^{4}$ and $\mathbb{S}^{1} \times \mathbb{S}^{3}$ (which is not an Einstein manifold) have trivial topological invariants. However, the stability of these product manifolds may be guaranteed by a lower-dimensional topology.
}

An underlying idea is that gravity can be formulated as a gauge theory of the Lorentz group where spin connections play a role of gauge fields and Riemann curvature tensors correspond to their field strengths [19]. Another important point is that Riemann curvature tensors $R_{b}^{a}$ are spin(4)-valued 2-forms in $\Omega^{2}(M)=$ $\Lambda^{2} T^{*} M$. These facts are combined with the well-known theorems (see Chap. 13 in Ref. [7] and Chaps. 1 and 2 in Ref. [8]).

\section{A. Self-duality}

On an orientable Riemannian four-manifold, the 2-forms decompose into the space of self-dual and anti-self-dual 2forms:

$$
\Omega^{2}=\Omega_{+}^{2} \oplus \Omega_{-}^{2}
$$

defined by the \pm 1 eigenspaces of the Hodge star operator $*: \Omega^{2} \rightarrow \Omega^{2}$.

\section{B. Lie group isomorphism}

There is a global isomorphism between the fourdimensional Lorentz group and classical Lie group, i.e., $S O(4)=S U(2)_{+} \otimes S U(2)_{-} / \mathbb{Z}_{2} \quad$ or $\quad \operatorname{Spin}(4)=S U(2)_{+}$ $\otimes S U(2)_{-}$. It also leads to the splitting of the Lie algebra

$$
s o(4)=s u_{+}(2) \oplus s u_{-}(2) .
$$

A central point is that these two decompositions are deeply related to each other due to the canonical vector space isomorphism between the Clifford algebra $\mathbb{C l}(4)$ in four dimensions and the exterior algebra $\Omega^{*} M=$ $\bigoplus_{k=0}^{4} \Lambda^{k} T^{*} M$ over a four-dimensional Riemannian manifold $M$ (see Chap. 2 in Ref. [20]). For the isomorphism between the vector spaces, the chiral operator $\gamma_{5}=$ $-\gamma_{1} \gamma_{2} \gamma_{3} \gamma_{4}$ in the Clifford algebra corresponds to the Hodge-dual operator $*: \Omega^{k} \rightarrow \Omega^{4-k}$ in the exterior algebra. Indeed, the splitting of vector spaces is induced by the existence of the projection operators

$$
P_{ \pm}=\frac{1}{2}(1 \pm *), \quad P_{ \pm}=\frac{1}{2}\left(1 \pm \gamma_{5}\right)
$$

acting on the vector space $\Omega^{2}$ and the so(4) generators $J_{a b}=\frac{1}{4}\left[\gamma_{a}, \gamma_{b}\right]$, respectively. [See Appendix A for the explicit matrix representations of so(4) Lie algebra.] Therefore, the splitting of the two vector spaces in Eqs. (2.4) and (2.5) is isomorphic to each other.

Thus, one can apply these decompositions to spin connections and curvature tensors $[9,10,21]$. The first decomposition is that the spin connections can be split into a pair of $S U(2)_{+}$and $S U(2)_{-}$gauge fields according to the Lie algebra splitting (2.5): 


$$
\omega_{a b}=A^{(+) i} \eta_{a b}^{i}+A^{(-) i} \bar{\eta}_{a b}^{i}
$$

where $\eta_{a b}^{i}$ and $\bar{\eta}_{a b}^{i}$ are the 't Hooft symbols satisfying the self-duality relation

$$
\eta_{a b}^{i}=\frac{1}{2} \varepsilon_{a b c d} \eta_{c d}^{i}, \quad \bar{\eta}_{a b}^{i}=-\frac{1}{2} \varepsilon_{a b c d} \bar{\eta}_{c d}^{i} .
$$

Note that the index $i=(1,2,3)$ refers to the $s u(2)_{ \pm}$ Lie algebra index. Appendix A contains the explicit representation of $s o(4)$ Lie algebra and the 't Hooft symbols. Accordingly the Riemann curvature tensors are also decomposed into a pair of $S U(2)_{+}$and $S U(2)_{-}$field strengths:

$$
R_{a b}=F^{(+) i} \eta_{a b}^{i}+F^{(-) i} \bar{\eta}_{a b}^{i}
$$

where $S U(2)_{ \pm}$field strengths are 2-forms on $M$ defined by

$$
\begin{aligned}
F^{( \pm) i} & =\frac{1}{2} F_{c d}^{( \pm) i} e^{c} \wedge d^{d} \\
& =d A^{( \pm) i}-\varepsilon^{i j k} A^{( \pm) j} \wedge A^{( \pm) k}
\end{aligned}
$$

The second decomposition (2.4) is that the sixdimensional vector space of 2-forms canonically splits into the sum of three-dimensional vector spaces of self-dual and anti-self-dual 2-forms. Canonical bases of self-dual and anti-self-dual 2-forms are given by

$$
\zeta_{+}^{i}=\frac{1}{2} \eta_{a b}^{i} e^{a} \wedge e^{b}, \quad \zeta_{-}^{i}=\frac{1}{2} \bar{\eta}_{a b}^{i} e^{a} \wedge e^{b}
$$

Using these bases, one can decompose the $S U(2)_{ \pm}$field strengths in Eq. (2.10) as

$$
F^{(+) i}=f_{(++)}^{i j} \zeta_{+}^{j}+f_{(+-)}^{i j} \zeta_{-}^{j}, \quad F^{(-) i}=f_{(-+)}^{i j} \zeta_{+}^{j}+f_{(--)}^{i j} \zeta_{-}^{j},
$$

where the canonical bases in Eq. (2.11) satisfy the Hodgeduality equation

$$
* \zeta_{ \pm}^{i}= \pm \zeta_{ \pm}^{i}
$$

Combining the two decompositions (2.9) and (2.12) leads to an irreducible decomposition of the general Riemann curvature tensor $[6,7,9,10]$ :

$$
\begin{aligned}
R_{a b c d}= & f_{(++)}^{i j} \eta_{a b}^{i} \eta_{c d}^{j}+f_{(+-)}^{i j} \eta_{a b}^{i} \bar{\eta}_{c d}^{j}+f_{(-+)}^{i j} \bar{\eta}_{a b}^{i} \eta_{c d}^{j} \\
& +f_{(--)}^{i j} \bar{\eta}_{a b}^{i} \bar{\eta}_{c d}^{j}
\end{aligned}
$$

The torsion-free condition (2.2) leads to an integrability condition, the so-called first Bianchi identity

$$
R_{a b c d}+R_{a c d b}+R_{a d b c}=0 .
$$

From the first Bianchi identity (2.15), one can derive the symmetry property

$$
R_{a b c d}=R_{c d a b} \text {. }
$$

Equation (2.16), being totally 15 conditions, imposes the symmetry property

$f_{(++)}^{i j}=f_{(++)}^{j i}, \quad f_{(--)}^{i j}=f_{(--)}^{j i}, \quad f_{(+-)}^{i j}=f_{(-+)}^{j i}$.

The first Bianchi identity (2.15), being totally 16 conditions, imposes an additional constraint

$$
f_{(++)}^{i j} \delta^{i j}=f_{(--)}^{i j} \delta^{i j}
$$

that is equivalently written as

$$
\varepsilon^{a b c d} R_{a b c d}=0
$$

If $(M, g)$ is an Einstein manifold satisfying the equations $R_{\mu \nu}=\lambda g_{\mu \nu}$ with $\lambda$ a cosmological constant, one can show (6.32 in Ref. [7] and Refs. [9,10]) that

$$
f_{(+-)}^{i j}=0=f_{(-+)}^{i j}
$$

In this case, the Riemann curvature tensor (2.9) is a direct sum of self-dual $S U(2)_{+}$field strengths and anti-self-dual $S U(2)$ _ field strengths taking the form

$$
F^{(+) i}=f_{(++)}^{i j} \zeta_{+}^{j}, \quad F^{(-) i}=f_{(--)}^{i j} \zeta_{-}^{j} .
$$

This means that $S U(2)_{ \pm}$field strengths describing an Einstein manifold correspond to Yang-Mills instantons obeying the self-duality equations explicitly written as

$$
F_{\mu \nu}^{( \pm) i}= \pm \frac{1}{2} \frac{\varepsilon^{\alpha \beta \rho \sigma}}{\sqrt{g}} g_{\mu \alpha} g_{\nu \beta} F_{\rho \sigma}^{( \pm) i},
$$

where $F^{( \pm) i}=\frac{1}{2} F_{\mu \nu}^{( \pm) i} d x^{\mu} \wedge d x^{\nu}=\frac{1}{2} F_{a b}^{( \pm) i} e^{a} \wedge e^{b}$. Therefore, an Einstein manifold $(M, g)$ has a configuration consisting of $S U(2)_{+}$Yang-Mills instantons and $S U(2)_{-}$ anti-instantons [9].

Since Einstein manifolds encode a topological information in the form of Yang-Mills instantons, it is natural to expect that the topological invariants of an Einstein manifold $(M, g)$ will be determined by the configuration of $S U(2)_{ \pm}$Yang-Mills instantons. For a general closed Riemannian manifold $M$, the Euler characteristic $\chi(M)$ and the Hirzebruch signature $\tau(M)$ are defined by $[7,14]$

$$
\begin{gathered}
\chi(M)=\frac{1}{32 \pi^{2}} \int_{M} \varepsilon^{a b c d} R_{a b} \wedge R_{c d}, \\
\tau(M)=\frac{1}{24 \pi^{2}} \int_{M} R_{a b} \wedge R_{a b} .
\end{gathered}
$$


The topological invariants can be expressed in terms of $S U(2)_{ \pm}$gauge fields using the decompositions (2.9) and (2.12)

$$
\begin{aligned}
\chi(M) & =\frac{1}{4 \pi^{2}} \int_{M}\left(F^{(+) i} \wedge F^{(+) i}-F^{(-) i} \wedge F^{(-) i}\right) \\
& =\frac{1}{2 \pi^{2}} \int_{M}\left(\left(f_{(++)}^{i j}\right)^{2}+\left(f_{(--)}^{i j}\right)^{2}-2\left(f_{(+-)}^{i j}\right)^{2}\right) \sqrt{g} d^{4} x, \\
\tau(M) & =\frac{1}{6 \pi^{2}} \int_{M}\left(F^{(+) i} \wedge F^{(+) i}+F^{(+) i} \wedge F^{(+) i}\right) \\
& =\frac{1}{3 \pi^{2}} \int_{M}\left(\left(f_{(++)}^{i j}\right)^{2}-\left(f_{(--)}^{i j}\right)^{2}\right) \sqrt{g} d^{4} x,
\end{aligned}
$$

where we used the volume element

$$
\zeta_{ \pm}^{i} \wedge \zeta_{ \pm}^{j}= \pm 2 \delta^{i j} \sqrt{g} d^{4} x, \quad \zeta_{ \pm}^{i} \wedge \zeta_{\mp}^{j}=0 .
$$

An Einstein manifold has curvature tensors given by Eq. (2.21) with the coefficients satisfying Eq. (2.18). In this case, the Euler characteristic $\chi(M)$ is given by the sum of self-dual and anti-self-dual instantons, whereas the Hirzebruch signature $\tau(M)$ is their difference. The above expression immediately verifies the famous inequalities for the topological invariants. The first inequality is $\chi(M) \geq 0$ with equality only if $f_{(++)}^{i j}=f_{(--)}^{i j}=0$, i.e., $M$ is flat (6.32 in Ref. [7] and Sec. 10.4 in [14]). The second inequality is the Hitchin-Thorpe inequality [22] stating that

$$
\chi(M) \pm \frac{3}{2} \tau(M)=\frac{1}{\pi^{2}} \int_{M}\left(f_{( \pm \pm)}^{i j}\right)^{2} \sqrt{g} d^{4} x \geq 0,
$$

where the equality holds only if $f_{(++)}^{i j}=0$ or $f_{(--)}^{i j}=0$, i.e., $M$ is half-flat (a gravitational instanton).

The instanton number for $S U(2)_{ \pm}$gauge fields is defined by ${ }^{2}$

$$
I^{( \pm)}= \pm \frac{1}{4 \pi^{2}} \int_{M} F^{( \pm) i} \wedge F^{( \pm) i}
$$

Then the topological invariants are determined by $S U(2)_{ \pm}$ instantons

$\chi(M)=\left(I^{(+)}+I^{(-)}\right) \geq 0, \quad \tau(M)=\frac{2}{3}\left(I^{(+)}-I^{(-)}\right)$.

\footnotetext{
${ }^{2}$ This definition has considered the fact [21] that $S U(2)_{ \pm}$ gauge fields from spin connections in Eq. (2.7) are related to Yang-Mills gauge fields by $A_{G}^{i}=-\frac{1}{2} A_{\mathrm{YM}}^{i}$ and $F_{G}^{i}=-\frac{1}{2} F_{\mathrm{YM}}^{i}$ and $S U(2)$ generators in gravity and gauge theory are related by $T_{G}^{i}=-2 \tau_{\mathrm{YM}}^{i}$. Note that the $4 \times 4$ matrices $T_{G}^{i}=\eta_{a b}^{i}$ or $\bar{\eta}_{a b}^{i}$ correspond to the spin $s=\frac{3}{2}$ representation of $S U(2)$ Lie algebra as shown in Eqs. (A7) and (A8), while the $2 \times 2$ Pauli matrices $\tau^{i}=2 i \tau_{\mathrm{YM}}^{i}$ in $S U(2)$ gauge fields are the spin $s=\frac{1}{2}$ representation.
}

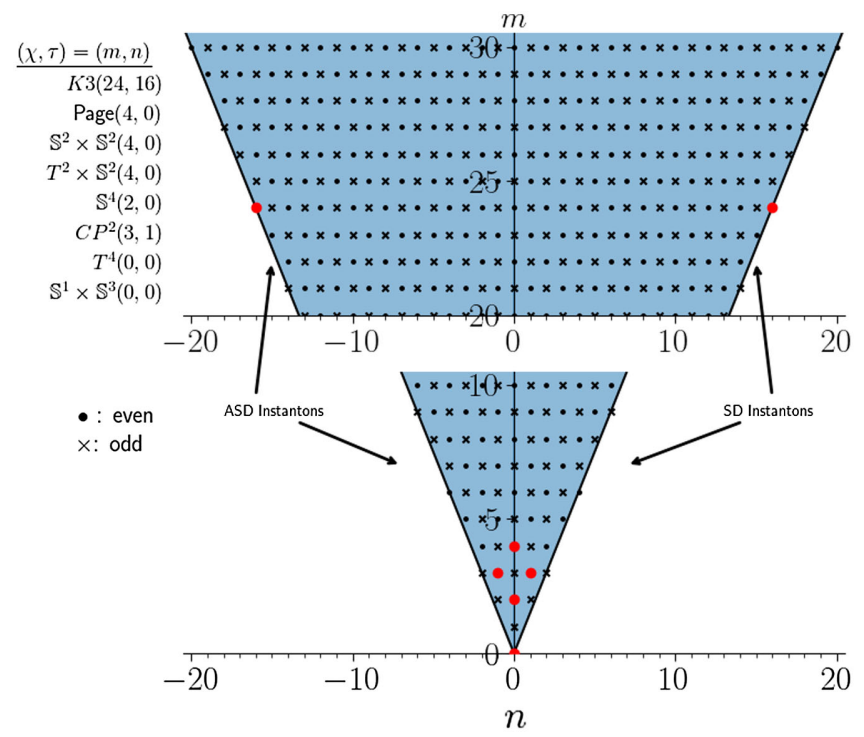

FIG. 1. Topological numbers of closed Einstein manifolds.

Let $\chi(M)=m \in \mathbb{Z}_{\geq 0}$ and $\tau(M)=n \in \mathbb{Z}$. We can invert Eq. (2.29) as

$$
I^{( \pm)}=\frac{1}{4}(2 m \pm 3 n)
$$

Note that our sign convention in Eq. (2.28) is $I^{( \pm)} \geq 0$, so the relation (2.30) is consistent with the inequality (2.27). The above relations show how the topology of Einstein manifolds is characterized by the configuration of $S U(2)_{+}$ instantons and $S U(2)_{-}$anti-instantons. One can also deduce that $\chi(M)+\tau(M)=m+n=2\left(1-b_{1}+b_{2}^{+}\right) \in 2 \mathbb{Z}$, where $b_{i}=\operatorname{dim} H^{i}(M, \mathbb{R})$ is the $i$ th Betti number (Chap. 6.D in Ref. [7] and Sec. 10.4 in Ref. [14]). This means that the set $(m, n)$ of topological numbers forms an even integer lattice, i.e., $m+n \in 2 \mathbb{Z}$. Some examples of four-dimensional compact Einstein manifolds are shown in Fig. 1, where the structure of the inverted triangle for an allowed region is due to the inequalities $m \geq 0$ and $m \geq \frac{3}{2}|n|$. In the list in Fig. 1, $\mathbb{S}^{1} \times \mathbb{S}^{3}$ is not an Einstein manifold, since it does not admit Einstein metrics [22] and Page is an inhomogeneous Einstein metric on the product of the nontrivial $\mathbb{S}^{2}$ bundle over $\mathbb{S}^{2}$ [23].

Figure 1 clearly shows the "reflection" symmetry [11]. The reflection symmetry can be realized by considering two compact Einstein manifolds $(M, g)$ and $(\tilde{M}, \tilde{g})$ obeying the following relation:

$$
I^{(+)}(M)=I^{(-)}(\tilde{M}), \quad I^{(-)}(M)=I^{(+)}(\tilde{M}) .
$$

Under the above transformation (2.31), the topological invariants are related as

$$
\chi(M)=\chi(\tilde{M}), \quad \tau(M)=-\tau(\tilde{M}) .
$$


Thus, the reflection symmetry corresponds to the interchange of instantons and anti-instantons which is achieved by a change of the manifold's orientation. This map indicates that a four-manifold with $\tau(M)=0$ is self-mirror.

We take the Lie algebra generators of $S O(4)$ as

$$
J_{i j} \equiv \varepsilon_{i j k} J_{k}, \quad J_{i 4} \equiv N_{i},
$$

where $i, j, k=1,2,3$ and

$$
\begin{aligned}
J_{i} & =\frac{i}{2}\left(\tau^{i} \otimes \mathbf{1}_{2}\right)=\frac{i}{2}\left(\begin{array}{cc}
\tau^{i} & 0 \\
0 & \tau^{i}
\end{array}\right), \\
N_{i}=\frac{i}{2}\left(\tau^{i} \otimes \tau^{3}\right) & =\frac{i}{2}\left(\begin{array}{cc}
\tau^{i} & 0 \\
0 & -\tau^{i}
\end{array}\right) .
\end{aligned}
$$

They satisfy the commutation relations

$$
\begin{aligned}
{\left[J_{i}, J_{j}\right] } & =-\varepsilon^{i j k} J_{k}, \quad\left[N_{i}, N_{j}\right]=-\varepsilon^{i j k} J_{k}, \\
{\left[J_{i}, N_{j}\right] } & =-\varepsilon^{i j k} N_{k} .
\end{aligned}
$$

In this representation, the generators in the Cartan subalgebra are $-i J_{3}$ and $-i N_{3}$. An irreducible representation (irrep) of $S O(4)$ is labeled by the highest weight defined by these operators [24], which is denoted by a state

$$
\left|\frac{p}{2}, \frac{q}{2}\right\rangle, \quad p \geq|q|,
$$

where $p$ and $q$ are both even integers or both odd integers. The isomorphism $\operatorname{Spin}(4) \cong S U(2)_{+} \otimes S U(2)_{-}$can be realized by taking

$$
J_{i}^{(+)} \equiv \frac{1}{2}\left(J_{i}+N_{i}\right), \quad J_{i}^{(-)} \equiv \frac{1}{2}\left(J_{i}-N_{i}\right),
$$

because they separately obey the $s u(2) \cong s o(3)$ commutation relations

$$
\left[J_{i}^{( \pm)}, J_{j}^{( \pm)}\right]=-\varepsilon^{i j k} J_{k}^{( \pm)}, \quad\left[J_{i}^{( \pm)}, J_{j}^{(\mp)}\right]=0 .
$$

For each $S U(2)_{ \pm}$factor, one may take a spin- $j_{ \pm}$representation such that $J_{i}^{( \pm)} J_{i}^{( \pm)}=-j_{ \pm}\left(j_{ \pm}+1\right)$. We choose $j_{+} \geq j_{-}$. An $S O(4)$ irrep in this basis is then labeled by a pair of integers or half integers $\left(j_{+}, j_{-}\right)$, i.e., the angular momenta associated with the $s u(2)_{+}$and $s u(2)_{-}$subalgebras. We denote the highest weight state as

$$
\left|j_{+}, j_{-}\right\rangle \text {. }
$$

The two representations are related by putting $p=2\left(j_{+}+\right.$ $\left.j_{-}\right)$and $q=2\left(j_{+}-j_{-}\right)$(Sec. 19.13 in Ref. [24]). The irrep of the direct product $\mathcal{D}\left(j_{+}\right) \otimes \mathcal{D}\left(j_{-}\right)$is decomposed as

$$
\left[\frac{p}{2}, \frac{q}{2}\right] \rightarrow\left[\frac{p}{2}\right] \oplus\left[\frac{p}{2}-1\right] \oplus \cdots \oplus\left[\frac{|q|}{2}\right]
$$

under the restriction $S O(4) \rightarrow S O(3)$.

We note that the separation of instantons such as Eq. (2.21) is caused by the splitting of the Lie algebra (2.5). Considering the fact that the instanton action $S=$ $8 \pi^{2}\left|I^{( \pm)}\right|$is determined by the instanton number itself, it may be reasonable to identity the instanton numbers $I^{( \pm)}$ with the labels characterizing some irreps of $S U(2)_{ \pm}$. Now we identify the labels $\left(j_{+}, j_{-}\right)$in the representation (2.39) with the instanton numbers in Eq. (2.30) as follows:

$$
\begin{aligned}
& j_{+}=2 I^{(+)}=\frac{1}{2}(2 m+3 n) \geq 0, \\
& j_{-}=2 I^{(-)}=\frac{1}{2}(2 m-3 n) \geq 0 .
\end{aligned}
$$

This identification automatically satisfies the HitchinThorpe inequality (2.27). However, in order to satisfy the condition that the set $(m, n)$ of topological numbers forms an even integer lattice, i.e., $m+n \in 2 \mathbb{Z}$, it is necessary to choose $\left(j_{+}, j_{-}\right)$such that both are integers or half integers and $5 j_{+}+j_{-} \in 12 \mathbb{Z}$. This identification leads to the identification $p=2\left(j_{+}+j_{-}\right)=4 m=4 \chi \geq 0$ and $q=2\left(j_{+}-j_{-}\right)=6 n=6 \tau$ in the representation (2.36). The condition $m+n \in 2 \mathbb{Z}$ corresponds to the requirement that both $p$ and $q$ are even and $3 p+$ $2 q \in 24 \mathbb{Z}$. The reflection symmetry (2.31) corresponds to the interchange of the representations, $\left(j_{+} \leftrightarrow j_{-}\right)$, under which $(p, q) \rightarrow(p,-q)$. This is the reason why it is enough to consider only the case, $j_{+} \geq j_{-}$, i.e., $q \geq 0$. Note that the representations $\mathcal{D}\left(j_{+}\right) \otimes \mathcal{D}\left(j_{-}\right)$and $\mathcal{D}\left(j_{-}\right) \otimes$ $\mathcal{D}\left(j_{+}\right)$for $j_{+} \neq j_{-}$correspond to distinct representations in $S O(4)$. It may be pointed out that the identification (2.41) does not explain the Hitchin-Thorpe inequality (2.27), because we have chosen the representations $\left(j_{+}, j_{-}\right)$such that they obey the relation $j_{+}=2 I^{(+)}$and $j_{-}=2 I^{(-)}$. Nevertheless, it is very encouraging that it is always possible to choose the $S O(4)$ representations so that such a relation is satisfied.

The four-dimensional Lorentz group Spin(4) is the spin group in dimension 4 , the double cover of $S O(4)$, that is a product group since $\operatorname{Spin}(4)=S U(2)_{+} \times S U(2)_{-}$and its Lie algebra becomes a direct sum of two $s u(2)_{ \pm}$Lie algebras. The splitting of the Lie algebra in Eq. (2.5) is related to the decomposition of the 2-forms on a fourmanifold in Eq. (2.4). The canonical splitting of the vector spaces occupies a central position for the instanton structure of Einstein manifolds. However, one may think of the four-dimensional gravity as being obtained through the Kaluza-Klein reduction of a five-dimensional gravity. Then one can consider the four-dimensional Einstein manifolds as obtained from a five-dimensional gravitational solution. The five-dimensional Lorentz group is $S O(5)$ which is a 
simple group. Since $S O(4) \subset S O(5)$, it will be interesting to see how the instanton configuration of Einstein manifolds fits into a multiplet in the irrep of $S O(5)$. We will discuss this issue in the next section.

\section{REPRESENTATION OF RIEMANNIAN MANIFOLDS}

As we have observed in Sec. II, the separation of Riemann curvature tensors has been originated from the splitting of the Lie algebra vector space (2.5). Since the vector space so(4) is isomorphic to the vector space of 2-forms $\Omega^{2}$, the same kind of splitting must also arise in the vector space $\Omega^{2}$. Equation (2.4) precisely shows such a splitting of the vector space $\Omega^{2}$. The gravitational force is represented by Riemann curvature tensors, and Einstein manifolds are described by two independent components of Riemann tensors (i.e., self-dual and anti-self-dual gravitational instantons). ${ }^{3}$ However, an interesting physics arises if we consider the four-dimensional gravity as being obtained from a five-dimensional gravity through the Kaluza-Klein compactification [12]. Then the fivedimensional Lorentz group is $S O(5)$ that is a simple group unlike the group $S O(4)=S U(2)_{+} \times S U(2)_{-} / \mathbb{Z}_{2}$. Moreover, there is no concept of self-duality for 2-forms in five dimensions so that the vector space $\Omega^{2}$ is no more decomposed. Therefore, neither the Lie algebra of $S O(5)$ nor the vector space of 2 -forms $\Omega^{2}$ is split in five dimensions. This implies that the self-dual and anti-selfdual components in $S U(2)_{ \pm}$factors must be combined in five dimensions, since the group $S O(4)=S U(2)_{+} \times$ $S U(2)_{-} / \mathbb{Z}_{2}$ has to be embedded into the simple group $S O(5)$. In other words, $S U(2)_{+}$instantons and $S U(2)_{-}$ anti-instantons in four-dimensional Einstein manifolds must appear in the same multiplet of the Lorentz group $S O(5)$. Therefore, the five-dimensional Kaluza-Klein theory unifies two independent sectors of curvature tensors as well as the electromagnetic force into a single gravitational force.

This scheme is similar to the grand unification of the Standard Model, although the Kaluza-Klein theory is defined in five-dimensional space. The Standard Model has a product gauge group $S U(3) \times S U(2) \times U(1)$ to describe the electroweak and strong forces. In the GUT, the Standard Model gauge group is embedded into a single gauge group, for example, $S U(5)$ or $S O(10)$. Then the leptons and quarks appear in the same multiplet in a larger

\footnotetext{
${ }^{3}$ It is not the case for the Lorentzian signature, because the local Lorentz group $S O(3,1)$ is a simple group although it is not compact. Furthermore, it does not mean that there are two independent gravitational forces, because the gravitational force is transmitted by metrics (not connections) and the metric does not decompose in any sense into a sum of two independent parts. The self-dual and anti-self-dual components of spin connections are described by the same metric.
}

symmetry [13]. Now we will see how four-dimensional Riemannian manifolds are similarly combined into a fivedimensional Einstein manifold. Furthermore, we will see that $S U(2)_{+}$instantons and $S U(2)_{-}$anti-instantons play a role of quarks and antiquarks from the point of view of a five-dimensional Einstein manifold. In order to analyze the anatomy of Riemannian manifolds, we will greatly use the group isomorphism [2]

$$
S O(5) \cong S p(2) / \mathbb{Z}_{2}
$$

We provide more details about the Lie algebras $s o(4)=$ $s u(2)_{+} \oplus s u(2)_{-}$and $s o(5) \cong s p(2)$ in Appendix A.

Let $N$ be a five-dimensional Riemannian manifold whose metric is given by

$$
d s^{2}=G_{M N}(X) d X^{M} d X^{N} .
$$

Introduce at each spacetime point on $N$ a local basis of orthonormal tangent vectors $\mathcal{E}_{A}=\mathcal{E}_{A}^{M} \partial_{M} \in \Gamma(T N)$ and its dual basis $E^{A}=E_{M}^{A} d X^{M} \in \Gamma\left(T^{*} N\right)$ defined by a natural pairing $\left\langle E^{A}, \mathcal{E}_{B}\right\rangle=\delta_{B}^{A}$, where $A, B=1, \ldots, 5 ; M, N=1$, $\ldots, 5$. In terms of the noncoordinate basis in $\Gamma\left(T^{*} N\right)$, the metric (3.2) can be written as

$$
d s^{2}=G_{M N}(X) d X^{M} d X^{N}=\delta_{A B} E^{A} \otimes E^{B} .
$$

Let us consider Einstein manifolds $(N, G)$ described by the Einstein-Hilbert action

$$
S_{5}=-\frac{1}{16 \pi G_{5}} \int(R-3 \Lambda) \sqrt{G} d^{5} X,
$$

where $\Lambda$ and $G_{5}$ are a cosmological constant and the gravitational constant in five dimensions, respectively. The equations of motion derived from the action (3.4) are

$$
R_{M N}=\Lambda G_{M N}
$$

A solution to Eq. (3.5) constitutes five-dimensional Einstein manifolds $(N, G)$. Now we consider the KaluzaKlein compactification of five-dimensional Einstein manifolds by assuming that the five-dimensional space $N$ is a cylinder $M \times \mathbb{S}^{1}$ with $0 \leq x^{5} \leq L=2 \pi R_{5}$ [12]. We split five-dimensional coordinates as $X^{M}=\left(x^{\mu}, x^{5}\right), \mu=1, \ldots, 4$, according to the cylinder geometry. Then the fivedimensional metric tensor in (3.2) also splits into fourdimensional fields, $g_{\mu \nu}(x), A_{\mu}(x)$, and $\phi(x)$. We have imposed the cylinder condition that the fields should not depend on the fifth coordinate $x^{5}$. We take the Kaluza-Klein ansatz for the five-dimensional metric in the form

$$
\begin{aligned}
d s^{2} & =G_{M N}(X) d X^{M} d X^{N} \\
& =e^{-\frac{1}{3} \phi}\left(g_{\mu \nu} d x^{\mu} d x^{\nu}+e^{\phi}\left(d x^{5}+\kappa A_{\mu} d x^{\mu}\right)^{2}\right),
\end{aligned}
$$


where $\kappa^{2}=16 \pi G_{4}$ and $G_{4}=\frac{G_{5}}{L}$ is the four-dimensional gravitational constant. It may be instructive to write the five-dimensional metric in the matrix form

$$
G_{M N}=e^{-\frac{1}{3} \phi}\left(\begin{array}{cc}
g_{\mu \nu}+\kappa^{2} e^{\phi} A_{\mu} A_{\nu} & \kappa e^{\phi} A_{\mu} \\
\kappa e^{\phi} A_{\nu} & e^{\phi}
\end{array}\right) .
$$

The geometric details of the five-dimensional gravity and the Kaluza-Klein theory appear in Appendix B.

Using the result (B21), one can write down the fivedimensional Einstein-Hilbert action (3.4) for the KaluzaKlein ansatz (3.6). First note that the five-dimensional volume form is $\sqrt{G} d^{5} X=E^{1} \wedge \cdots \wedge E^{5}=e^{-\frac{1}{3} \phi} \sqrt{g} d^{4} x d x^{5}$. Since the four-dimensional fields do not depend on the circle coordinate $x^{5}$, one can integrate out the fifth coordinate that gives rise to the redefinition of the gravitational constant $G_{4}=\frac{G_{5}}{L}$. Moreover, one can ignore the Laplacian term in Eq. (B21) because it becomes a boundary term. Finally, the Einstein-Hilbert action (3.4) reduces to the four-dimensional action

$$
\begin{aligned}
S= & \int\left(-\frac{1}{16 \pi G_{4}}\left({ }^{(4)} R-3 e^{-\frac{\kappa}{\sqrt{3}} \Phi} \Lambda\right)\right. \\
& \left.+\frac{1}{4} e^{\sqrt{3} \kappa \Phi} g^{\mu \rho} g^{\nu \sigma} F_{\mu \nu} F_{\rho \sigma}+\frac{1}{2} g^{\mu \nu} \partial_{\mu} \Phi \partial_{\nu} \Phi\right) \sqrt{g} d^{4} x,
\end{aligned}
$$

where we have rescaled the scalar field

$$
\Phi \equiv \frac{1}{\sqrt{3} \kappa} \phi
$$

such that the scalar field has the usual kinetic term with canonical mass dimension. The Ricci scalar ${ }^{(4)} R$ with the left-hand superscript ${ }^{(4)}$ is determined only by the fourdimensional metric $d s_{4}^{2}=g_{\mu \nu}(x) d x^{\mu} d x^{\nu}$. Note that $\Lambda$ no longer behaves like a cosmological constant in four dimensions except the case of a constant scalar field.

Let us consider the symmetries of the Kaluza-Klein geometry with the metric (3.6) where the components of the gravitational field along the circle transmute into the electromagnetic field. The effective field theory of five-dimensional gravity around a solution of the form $N=M \times \mathbb{S}^{1}$ is four-dimensional gravity coupled to electromagnetism and a dilaton field. The five-dimensional Lorentz transformations that would mix the remaining fourdimensional gravitational excitations with electromagnetic excitations are not symmetries of the metric. The symmetries of the Kaluza-Klein vacuum (3.6) are the fourdimensional Lorentz symmetries, acting on $M$, and a $U(1)$ group acting on the circle $\mathbb{S}^{1}[12,25]$. These symmetries are realized as local or gauge symmetries in the apparent fourdimensional world, because the whole theory started with the Einstein-Hilbert action (3.4) which is generally covariant. Therefore, the spontaneous symmetry breaking by the Kaluza-Klein ground state (3.6) arises via a two-step procedure with the symmetry breaking from $S O(5)$ to $S O(4)$ followed by the symmetry enhancement to $S O(4) \times$ $U(1)$ in terms of the isometry of the Kaluza-Klein circle. The remaining symmetry is denoted as

$$
S O(5) \rightarrow S O(4) \times U(1),
$$

although the $U(1)$ factor is not a subgroup of $S O(5)$, since it acts on the circle coordinate as

$$
x^{\mu} \mapsto x^{\mu}, \quad x^{5} \mapsto x^{5}+f(x) .
$$

Under this transformation, we have

$g_{\mu \nu} \mapsto g_{\mu \nu}, \quad \phi \mapsto \phi, \quad A_{\mu} \mapsto A_{\mu}-\frac{1}{\kappa} \partial_{\mu} f$,

so that the 1-form $A=A_{\mu} d x^{\mu}$ transforms like an Abelian gauge field.

The equations of motion for the four-dimensional fields can be derived from the action (3.8):

$$
\begin{aligned}
{ }^{(4)} R_{\mu \nu}-\frac{1}{2} g_{\mu \nu}\left({ }^{(4)} R-3 e^{-\frac{\kappa}{\sqrt{3}} \Phi} \Lambda\right) & =8 \pi G_{4} T_{\mu \nu}, \\
D_{\mu}\left(e^{\sqrt{3} \kappa \Phi} F^{\mu \nu}\right) & =0,
\end{aligned}
$$

$$
\Delta \Phi=\frac{\sqrt{3} \kappa}{4} e^{\sqrt{3} \kappa \Phi} F_{\mu \nu} F^{\mu \nu}-\frac{\sqrt{3}}{\kappa} e^{-\frac{\kappa}{\sqrt{3}} \Phi} \Lambda,
$$

where the energy-momentum tensor $T_{\mu \nu}$ is given by

$$
\begin{aligned}
T_{\mu \nu}= & e^{\sqrt{3} \kappa \Phi} g^{\rho \sigma} F_{\mu \rho} F_{\nu \sigma}+\partial_{\mu} \Phi \partial_{\nu} \Phi \\
& -g_{\mu \nu}\left(\frac{1}{4} e^{\sqrt{3} \kappa \Phi} F_{\rho \sigma} F^{\rho \sigma}+\frac{1}{2} g^{\rho \sigma} \partial_{\rho} \Phi \partial_{\sigma} \Phi\right) .
\end{aligned}
$$

Indeed, one can check using the results in Eqs. (B18)(B21) and $R=5 \Lambda$ that the above equations of motion are exactly the same as Eq. (3.5) for a five-dimensional Einstein manifold. Therefore, the general five-dimensional metric (3.6) describes a five-dimensional Einstein manifold as long as the four-dimensional fields, $g_{\mu \nu}(x), A_{\mu}(x)$, and $\Phi(x)$, satisfy the above equations of motion. It will be interesting to see how the other fields such as $A_{\mu}(x)$ and $\Phi(x)$ deform the instanton structure of four-dimensional Einstein manifolds and understand how these deformed geometries are nicely unified into a five-dimensional Einstein manifold.

In order to understand such a structure of a fivedimensional Einstein manifold, it would be useful to have the decomposition of five-dimensional Riemann curvature tensors similar to the four-dimensional decomposition 
(2.14). The generators of so(5) Lorentz algebra are defined by

$$
J_{A B}=\frac{1}{4}\left[\gamma_{A}, \gamma_{B}\right]
$$

and they satisfy the Lorentz algebra

$$
\left[J_{A B}, J_{C D}\right]=-\left(\delta_{A C} J_{B D}-\delta_{A D} J_{B C}-\delta_{B C} J_{A D}+\delta_{B D} J_{A C}\right) .
$$

See Appendix A for the representation of the fivedimensional gamma matrices. The ten generators in Eq. (3.17) consist of $J_{A B}=\left(J_{a b}, J_{5 a}\right)$, where the generators $J_{a b}$ satisfy the four-dimensional Lorentz algebra $s o(4) \subset$ so(5) and $J_{5 a}$ are additional generators given by

$$
\begin{aligned}
J_{a b} & =\frac{1}{4}\left[\gamma_{a}, \gamma_{b}\right]=\frac{i}{2}\left(\begin{array}{cc}
\eta_{a b}^{i} \tau^{i} & 0 \\
0 & \bar{\eta}_{a b}^{i} \tau^{i}
\end{array}\right), \\
J_{5 a}=\frac{1}{4}\left[\gamma_{5}, \gamma_{a}\right] & =\frac{1}{2}\left(\begin{array}{cc}
0 & \sigma^{a} \\
-\bar{\sigma}^{a} & 0
\end{array}\right) .
\end{aligned}
$$

Let us denote the generators in Eq. (3.19) as

$$
\begin{array}{ll}
J_{i j} \equiv \varepsilon_{i j k} T^{k}, & J_{i 4} \equiv T^{3+i}, \\
J_{i 5} \equiv-T^{6+i}, & J_{45} \equiv-T^{10},
\end{array}
$$

which take the block matrix form

$$
\begin{aligned}
T^{i} & =\frac{i}{2}\left(\tau^{i} \otimes \mathbf{1}_{2}\right)=\frac{i}{2}\left(\begin{array}{cc}
\tau^{i} & 0 \\
0 & \tau^{i}
\end{array}\right), \\
T^{3+i}=\frac{i}{2}\left(\tau^{i} \otimes \tau^{3}\right) & =\frac{i}{2}\left(\begin{array}{cc}
\tau^{i} & 0 \\
0 & -\tau^{i}
\end{array}\right), \\
T^{6+i}=\frac{i}{2}\left(\tau^{i} \otimes \tau^{1}\right) & =\frac{i}{2}\left(\begin{array}{cc}
0 & \tau^{i} \\
\tau^{i} & 0
\end{array}\right), \\
T^{10}=\frac{i}{2}\left(\mathbf{1}_{2} \otimes \tau^{2}\right) & =\frac{1}{2}\left(\begin{array}{cc}
0 & \mathbf{1}_{2} \\
-\mathbf{1}_{2} & 0
\end{array}\right) .
\end{aligned}
$$

It can be shown (see Appendix A) that the $4 \times 4$ matrices $T^{\AA}, \mathbb{A}=1, \ldots, 10$, in Eq. (3.21) constitute the Lie algebra generators of $s p(2)$. Therefore, we establish the Lie algebra isomorphism $s p(2) \cong s o(5)$. Since the universal covering group of $S O(5)$ is $S p(2)$, we get the group isomorphism (3.1).

Since the vector spaces generated by $J_{A B}$ and $T^{\mathbb{A}}$ are isomorphic to each other, there exists a linear relation between them:

$$
J_{A B}=\psi_{A B}^{\mathbb{A}} T^{\AA}, \quad T^{\mathbb{A}}=\frac{1}{2} \psi_{A B}^{\AA} J_{A B},
$$

where

$$
\psi_{A B}^{\AA}=-\operatorname{Tr}\left(J_{A B} T^{\AA}\right) .
$$

The psi symbol in (3.23) is the analog of the fourdimensional 't Hooft symbols in Eq. (A6), which explicitly presents the Lie algebra isomorphism $s o(5) \cong s p(2)$. Indeed, the matrix expression $\left(T^{\mathbb{A}}\right)_{A B} \equiv \psi_{A B}^{\mathbb{A}}$ provides the five-dimensional representation of $s p(2)$ Lie algebra as was shown in Eq. (A36). The Riemann curvature tensor $R=\frac{1}{2} R_{A B} J_{A B} \in C^{\infty}\left(\mathfrak{g} \otimes \Omega^{2}\right)$ carries two kinds of indices living in different vector spaces:

$R_{A B}=\frac{1}{2} R_{A B M N} d X^{M} \wedge d X^{N}=\frac{1}{2} R_{A B C D} E^{C} \wedge E^{D}$,

where the indices $(A, B)$ live in the vector space of $\mathfrak{g}=s o(5)$ Lie algebra while $(C, D)$ live in the vector space of 2-forms $\Omega^{2}=\Lambda^{2} T^{*} N$. But these two vector spaces are isomorphic to each other, and their isomorphism is encoded in the symmetry property of curvature tensors:

$$
R_{A B C D}=R_{C D A B} .
$$

The symmetry property (3.25) can be derived from the first Bianchi identity

$$
R_{A B C D}+R_{A C D B}+R_{A D B C}=0,
$$

which is the integrability condition for the torsion 2-forms in Eq. (B1) $[14,18]$. Thus, we can expand the curvature tensors $R_{A B C D}$ in the $s p(2)$ basis for both indices using the psi symbol (3.23) as

$$
R_{A B C D}=\Re_{\mathrm{AB}} \psi_{A B}^{\mathrm{A}} \psi_{C D}^{\mathbb{B}},
$$

where the expansion coefficients are symmetric, i.e.,

$$
\mathfrak{R}_{\mathrm{AB}}=\mathfrak{R}_{\mathbb{B A}}=\frac{1}{4} R_{A B C D} \psi_{A B}^{\mathbb{A}} \psi_{C D}^{\mathbb{B}}
$$

due to the property (3.25). The Bianchi identity (3.26) which is totally 50 conditions imposes five additional conditions

$$
d^{\mathrm{ABC} C} \mathfrak{R}_{\mathrm{AB}}=0
$$

in addition to the 45 conditions from Eq. (3.28), where the structure constants $d^{\mathrm{ABC}}$ are defined in Eq. (A26). The constraints (3.29) can be derived from Eq. (3.27) by contracting $\frac{1}{4} J_{A B} J_{C D}$ on both sides and applying the products (A27) and (A28). Then it results in two identities

$$
\begin{gathered}
\mathfrak{R}_{\mathrm{ABB}} \delta^{\mathrm{AB}}=\frac{1}{2} R, \\
d^{\mathrm{AB} E} \boldsymbol{R}_{\mathrm{AB}}=\frac{1}{4} \varepsilon_{A B C D E} R_{A B C D}=0,
\end{gathered}
$$


where $R$ is the Ricci scalar and Eq. (3.31) must vanish due to the Bianchi identity (3.26). It may also be checked by counting the number of independent Riemann curvature tensors. In five dimensions, the number of independent Riemann curvature tensors obeying the Bianchi identity (3.26) is 50. The number of $s p(2)$ curvature tensors obeying Eq. (3.28) is $55=100-45$, and then imposing the five constraints (3.29) leads to 50 independent components. See Appendix C for the group structure of Riemann curvature tensor.

The $s p(2) \cong s o(5)$ generators in Eq. (3.21) satisfy the commutation relations

$$
\begin{aligned}
& {\left[T^{i}, T^{j}\right]=\left[T^{3+i}, T^{3+j}\right]=\left[T^{6+i}, T^{6+j}\right]=-\varepsilon^{i j k} T^{k},} \\
& {\left[T^{i}, T^{3+j}\right]=-\varepsilon^{i j k} T^{3+k}, \quad\left[T^{i}, T^{6+j}\right]=-\varepsilon^{i j k} T^{6+k}, \quad\left[T^{3+i}, T^{6+j}\right]=-\delta^{i j} T^{10},} \\
& {\left[T^{i}, T^{10}\right]=0, \quad\left[T^{3+i}, T^{10}\right]=T^{6+i}, \quad\left[T^{6+i}, T^{10}\right]=-T^{3+i} .}
\end{aligned}
$$

Note that $T^{3}=\frac{i}{2}\left(\tau^{3} \otimes \mathbf{1}_{2}\right)$ and $T^{6}=\frac{i}{2}\left(\tau^{3} \otimes \tau^{3}\right)$ are diagonal matrices. Therefore, they constitute the set of the Cartan subalgebra for $s p(2) \cong s o(5)$ :

$$
\mathfrak{h}=\left\{H^{1}=-i T^{3}, H^{2}=-i T^{6}\right\}
$$

They correspond to $H^{1}=-i J_{3}$ and $H^{2}=-i N_{3}$, respectively, according to the notation (2.33). Thus, this representation contains the highest weight state (2.36). The remaining generators are chosen to satisfy the eigenvalue equations $[13,24]$

$$
\left[H^{i}, E^{\alpha}\right]=\alpha^{i} E^{\alpha},
$$

where $i=1,2$. The two-dimensional vector $\vec{\alpha}=\left(\alpha^{1}, \alpha^{2}\right)$ is called a root, and $E^{\alpha}$ is the corresponding ladder operator. We choose the ladder operators as follows:

$$
\begin{aligned}
& A_{ \pm}=\frac{1}{2}\left(T^{1} \pm i T^{2}+\left(T^{4} \pm i T^{5}\right)\right), \quad B_{ \pm}=\frac{1}{2}\left(T^{1} \pm i T^{2}-\left(T^{4} \pm i T^{5}\right)\right) \\
& C_{ \pm}=T^{7} \pm i T^{8}, \quad D_{ \pm}=T^{9} \pm i T^{10} .
\end{aligned}
$$

It may be useful to show the explicit matrix representation of the Cartan-Weyl basis:

$$
\begin{array}{rlrl}
H^{1} & =\frac{1}{2}\left(\begin{array}{cc}
\tau^{3} & 0 \\
0 & \tau^{3}
\end{array}\right), & H^{2}=\frac{1}{2}\left(\begin{array}{cc}
\tau^{3} & 0 \\
0 & -\tau^{3}
\end{array}\right), \quad A_{ \pm}=\frac{1}{2}\left(\begin{array}{cc}
\tau^{ \pm} & 0 \\
0 & 0
\end{array}\right), \quad B_{ \pm}=\frac{1}{2}\left(\begin{array}{cc}
0 & 0 \\
0 & \tau^{ \pm}
\end{array}\right), \\
C_{ \pm}=\frac{1}{2}\left(\begin{array}{cc}
0 & \tau^{ \pm} \\
\tau^{ \pm} & 0
\end{array}\right), & D_{ \pm}=\frac{i}{2}\left(\begin{array}{cc}
0 & \pm \mathbf{1}_{2}+\tau^{3} \\
\mp \mathbf{1}_{2}+\tau^{3} & 0
\end{array}\right),
\end{array}
$$

where $\tau^{ \pm}=i\left(\tau^{1} \pm i \tau^{2}\right)$. Then the commutation relations in Eq. (3.32) can be written in the Cartan-Weyl basis as

$$
\begin{aligned}
& {\left[H^{1}, A_{ \pm}\right]= \pm A_{ \pm}, \quad\left[H^{2}, A_{ \pm}\right]=0,} \\
& {\left[H^{1}, B_{ \pm}\right]=0, \quad\left[H^{2}, B_{ \pm}\right]= \pm B_{ \pm},} \\
& {\left[H^{1}, C_{ \pm}\right]= \pm C_{ \pm}, \quad\left[H^{2}, C_{ \pm}\right]= \pm C_{ \pm},} \\
& {\left[H^{1}, D_{ \pm}\right]= \pm D_{ \pm}, \quad\left[H^{2}, D_{ \pm}\right]=\mp D_{ \pm} .}
\end{aligned}
$$

Therefore, we identify the root vectors derived from the ladder generators

$$
\begin{array}{ll}
\vec{\alpha}_{A_{ \pm}}= \pm(1,0), & \vec{\alpha}_{B_{ \pm}}= \pm(0,1), \\
\vec{\alpha}_{C_{ \pm}}= \pm(1,1), & \vec{\alpha}_{D_{ \pm}}= \pm(1,-1),
\end{array}
$$

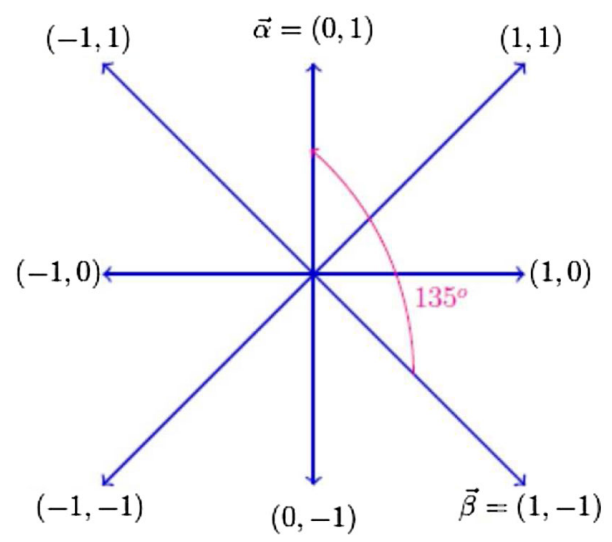

FIG. 2. Root diagram of $s o(5) \cong s p(2)$ for the Cartan subalgebra (3.33). 
where the first (second) entry of root vectors is the eigenvalue of $a d_{H^{1}}\left(a d_{H^{2}}\right)$. The corresponding root diagram is shown in Fig. 2. The simple roots are denoted by

$$
\vec{\alpha}=(0,1), \quad \vec{\beta}=(1,-1) .
$$

One may choose a different combination of the Cartan subalgebra

$$
\mathfrak{h}=\left\{H^{1}=-\frac{i}{\sqrt{2}}\left(T^{3}+T^{6}\right), H^{2}=-\frac{i}{\sqrt{2}}\left(T^{3}-T^{6}\right)\right\},
$$

whose matrix form is given by

$H^{1}=\frac{1}{\sqrt{2}}\left(\begin{array}{cc}\tau^{3} & 0 \\ 0 & 0\end{array}\right), \quad H^{2}=\frac{1}{\sqrt{2}}\left(\begin{array}{cc}0 & 0 \\ 0 & \tau^{3}\end{array}\right)$.

They correspond to $H^{1}=-\sqrt{2} i J_{3}^{(+)}$and $H^{2}=-\sqrt{2} i J_{3}^{(-)}$, respectively, according to the notation (2.37). Thus, this representation contains the highest weight state (2.39). Then the commutation relations in Eq. (3.32) can be written in the Cartan-Weyl basis as

$$
\begin{aligned}
& {\left[H^{1}, A_{ \pm}\right]= \pm \sqrt{2} A_{ \pm}, \quad\left[H^{2}, A_{ \pm}\right]=0} \\
& {\left[H^{1}, B_{ \pm}\right]=0, \quad\left[H^{2}, B_{ \pm}\right]= \pm \sqrt{2} B_{ \pm},} \\
& {\left[H^{1}, C_{ \pm}\right]= \pm \frac{1}{\sqrt{2}} C_{ \pm}, \quad\left[H^{2}, C_{ \pm}\right]= \pm \frac{1}{\sqrt{2}} C_{ \pm}} \\
& {\left[H^{1}, D_{ \pm}\right]= \pm \frac{1}{\sqrt{2}} D_{ \pm}, \quad\left[H^{2}, D_{ \pm}\right]=\mp \frac{1}{\sqrt{2}} D_{ \pm} .}
\end{aligned}
$$

The corresponding root diagram is shown in Fig. 3. The simple roots may be chosen as

$$
\vec{\alpha}=\sqrt{2}(0,1), \quad \vec{\beta}=\frac{1}{\sqrt{2}}(1,-1) .
$$

Our normalization for simple roots is that the square length of the longest roots is set equal to 2 .

As we have indicated in Eq. (3.24), the Riemann curvature tensors are 2-forms in $\Omega^{2}=\Lambda^{2} T^{*} N$ taking values in the vector space of $\mathfrak{g}=s o(5) \cong s p(2)$ Lie algebra. According to the remaining symmetry (3.10), let us decompose the Lie algebra $\mathfrak{g}=\operatorname{so}(5)=\left\{T^{\mathbb{A}} \mid \mathbb{A}=\right.$ $1, \ldots, 10\}$ as

$$
\mathfrak{g}=s o(4) \oplus \mathfrak{t}=s u(2)_{+} \oplus s u(2)_{-} \oplus \mathfrak{t},
$$

where $\mathfrak{f}$ contains the generators $J_{5 a}$ in the coset space $S O(5) / S O(4) \cong \mathbb{S}^{4}$. Since $\mathfrak{g} \cong \Lambda^{2} T^{*} N$ as vector spaces, Eq. (3.27) gives us the expansion of the curvature tensor in the basis of $\mathfrak{g}$. In the basis (3.20), $\left\{T^{\mathbb{A}} \mid \mathbb{A}=1, \ldots, 6\right\}$ corresponds to $J_{a b}$, so $s o(4) \subset \operatorname{so}(5)$ Lie algebra and $\left\{T^{6+a}: a=1, \ldots, 4\right\}$ corresponds to the coset generators $J_{5 a}$ in $\mathfrak{f}$. As is well known, Eq. (3.32) shows that the coset space $S O(5) / S O(4) \cong \mathbb{S}^{4}$ is reductive $([\operatorname{so}(4), \mathfrak{f}] \subset \mathfrak{f})$ and symmetric $([\mathfrak{x}, \mathfrak{f}] \subset \operatorname{so}(4))$. One can explicitly determine nonzero components of the psi symbols defined by Eq. (3.23) using Eq. (A27):

$\psi_{a b}^{i}=\varepsilon^{i a b 4}, \quad \psi_{a b}^{3+i}=\delta^{i a} \delta^{4 b}-\delta^{i b} \delta^{4 a}, \quad \psi_{5 b}^{6+a}=\delta^{a b}$.

Therefore, the 't Hooft symbols in Eq. (A6) are related to the psi symbols by

$$
\eta_{a b}^{i}=\psi_{a b}^{i}+\psi_{a b}^{3+i}, \quad \bar{\eta}_{a b}^{i}=\psi_{a b}^{i}-\psi_{a b}^{3+i} .
$$

Then the above combination implies that

$T^{i}+T^{3+i}=\frac{1}{2}\left(\psi_{a b}^{i}+\psi_{a b}^{3+i}\right) J_{a b}=\frac{1}{2} \eta_{a b}^{i} J_{a b} \in s u(2)_{+}$,

$T^{i}-T^{3+i}=\frac{1}{2}\left(\psi_{a b}^{i}-\psi_{a b}^{3+i}\right) J_{a b}=\frac{1}{2} \bar{\eta}_{a b}^{i} J_{a b} \in \operatorname{su}(2)_{-}$,

where we used the definition (3.22). The coset generators are given by

$$
T^{6+a}=\psi_{5 b}^{6+a} J_{5 b}=J_{5 a} \in \mathfrak{t}
$$

Thus, the Cartan-Weyl basis for the root diagram in Fig. 3 can be classified as follows:

$$
\left(H^{1}, A_{ \pm}\right) \in \operatorname{su}(2)_{+}, \quad\left(H^{2}, B_{ \pm}\right) \in \operatorname{su}(2)_{-}, \quad\left(C_{ \pm}, D_{ \pm}\right) \in \mathfrak{f} .
$$

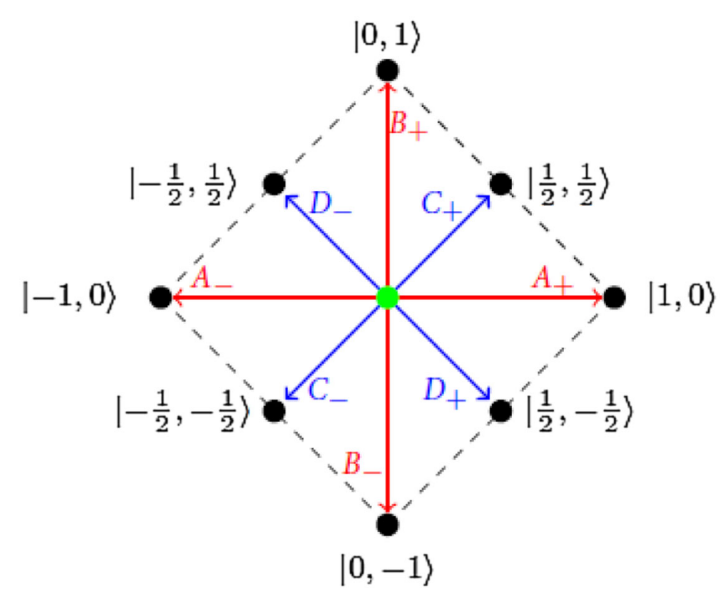

FIG. 3. The root diagram of $s p(2) \cong s o(5)$ for the Cartan subalgebra (3.40) where the actual roots must read as $\left|\alpha_{1}, \alpha_{2}\right\rangle=\sqrt{2}\left|\beta_{1}, \beta_{2}\right\rangle$. 
The decomposition of four-dimensional Einstein manifolds implies that $S U(2)_{+}$Yang-Mills instantons live in the vector space $s u(2)_{+}$and $S U(2)_{-}$Yang-Mills anti-instantons live in the vector space $s u(2)_{-}$. The root diagram in Fig. 3 shows how each component in the five-dimensional Riemann curvature tensors $R_{A B C D}$ deforms the instanton structure of four-dimensional Einstein manifolds.

Hence, it is useful to decompose the five-dimensional Riemann curvature tensors in Eq. (3.27) according to the Lie algebra decomposition (3.44). First, the Riemann curvature tensors in (B14) are decomposed as

$$
\begin{aligned}
R_{a b c d}= & \mathfrak{f}_{(++)}^{i j} \eta_{a b}^{i} \eta_{c d}^{j}+\mathfrak{f}_{(+-)}^{i j} \eta_{a b}^{i} \bar{\eta}_{c d}^{j}+\mathfrak{f}_{(-+)}^{i j} \bar{\eta}_{a b}^{i} \eta_{c d}^{j} \\
& +\mathfrak{f}_{(--)}^{i j} \bar{\eta}_{a b}^{i} \bar{\eta}_{c d}^{j},
\end{aligned}
$$

where

$$
\begin{aligned}
\mathfrak{f}_{(++)}^{i j} & \equiv \frac{1}{4}\left(\mathfrak{R}_{i, j}+\mathfrak{R}_{3+i, j}+\mathfrak{R}_{i, 3+j}+\mathfrak{R}_{3+i, 3+j}\right), \\
\mathfrak{f}_{(+-)}^{i j} & \equiv \frac{1}{4}\left(\mathfrak{R}_{i, j}+\mathfrak{R}_{3+i, j}-\mathfrak{R}_{i, 3+j}-\mathfrak{R}_{3+i, 3+j}\right), \\
\mathfrak{f}_{(-+)}^{i j} & \equiv \frac{1}{4}\left(\mathfrak{R}_{i, j}-\mathfrak{R}_{3+i, j}+\mathfrak{R}_{i, 3+j}-\mathfrak{R}_{3+i, 3+j}\right), \\
\mathfrak{f}_{(--)}^{i j} & \equiv \frac{1}{4}\left(\mathfrak{R}_{i, j}-\mathfrak{R}_{3+i, j}-\mathfrak{R}_{i, 3+j}+\mathfrak{R}_{3+i, 3+j}\right) .
\end{aligned}
$$

Explicitly, they are given by

$$
\begin{aligned}
\mathfrak{f}_{(++)}^{i j}= & e^{\frac{1}{3} \phi}\left\{f_{(++)}^{i j}\right. \\
& -\kappa^{2} e^{\phi}\left(\frac{3}{4} f^{(+) i} f^{(+) j}-\frac{1}{8}\left(f^{(+) k} f^{(+) k}-f^{(-) k} f^{(-) k}\right) \delta^{i j}\right) \\
& \left.+\frac{1}{24}\left(\Delta \phi-\frac{1}{6}\left(\partial_{a} \phi\right)^{2}\right) \delta^{i j}\right\}, \\
\mathfrak{f}_{(+-)}^{i j}= & e^{\frac{1}{3} \phi}\left\{f_{(+-)}^{i j}-\frac{3}{4} \kappa^{2} e^{\phi} f^{(+) i} f^{(-) j}\right. \\
& \left.+\frac{1}{24}\left(D_{a} \partial_{b} \phi+\frac{1}{6} \partial_{a} \phi \partial_{b} \phi\right) \eta_{a c}^{i} \bar{\eta}_{b c}^{j}\right\}, \\
\mathfrak{f}_{(--)}^{i j}= & e^{\frac{1}{3} \phi}\left\{f_{(--)}^{i j}\right. \\
& -\kappa^{2} e^{\phi}\left(\frac{3}{4} f^{(-) i} f^{(-) j}+\frac{1}{8}\left(f^{(+) k} f^{(+) k}-f^{(-) k} f^{(-) k}\right) \delta^{i j}\right) \\
& \left.+\frac{1}{24}\left(\Delta \phi-\frac{1}{6}\left(\partial_{a} \phi\right)^{2}\right) \delta^{i j}\right\},
\end{aligned}
$$

where $f_{(* *)}^{i j}$ are the expansion coefficients of the fourdimensional Riemann curvature tensors (2.14) and we have introduced a similar decomposition for $U(1)$ field strengths

$$
F_{a b}=f^{(+) i} \eta_{a b}^{i}+f^{(-) i} \bar{\eta}_{a b}^{i} .
$$

Note that

$$
\mathfrak{f}_{(++)}^{i j}=\mathfrak{f}_{(++)}^{j i}, \quad \mathfrak{f}_{(--)}^{i j}=\mathfrak{f}_{(--)}^{j i}, \quad \mathfrak{f}_{(+-)}^{i j}=\mathfrak{f}_{(-+)}^{j i}
$$

due to the symmetry property (3.25) and the Bianchi identity (3.26) further requires

$$
\mathfrak{f}_{(++)}^{i j} \delta^{i j}=\mathfrak{f}_{(--)}^{i j} \delta^{i j} .
$$

It is easy to check Eq. (3.55) using the above results. ${ }^{4}$ Using the decomposition (3.53), it is straightforward to calculate the $U(1)$ instanton density:

$$
\begin{aligned}
\rho_{U(1)} & =\frac{1}{64 \pi^{2}} \varepsilon^{a b c d} F_{a b} F_{c d} \\
& =\frac{1}{8 \pi^{2}}\left(f^{(+) k} f^{(+) k}-f^{(-) k} f^{(-) k}\right) .
\end{aligned}
$$

Using Eq. (3.45), the expansion (3.27) for the Riemann tensors $R_{5 a b c}$ can be written as

$$
\begin{aligned}
R_{5 a b c} & =\Re_{6+a, \mathbb{B}} \psi_{b c}^{\mathbb{B}} \\
& \equiv \mathbb{F}_{5 a}^{(+) i} \eta_{b c}^{i}+\mathbb{F}_{5 a}^{(-) i} \bar{\eta}_{b c}^{i},
\end{aligned}
$$

where $\mathbb{F}_{5 a}^{( \pm) i}=\frac{1}{2}\left(\mathfrak{R}_{6+a, i} \pm \mathfrak{R}_{6+a, 3+i}\right)$ are given by

$$
\begin{aligned}
\mathbb{F}_{5 a}^{(+) i}= & \frac{\kappa}{12} e^{\frac{5}{6} \phi}\left(6 D_{a}^{(+)} f^{(+) i}+7 f^{(+) i} \partial_{a} \phi-2 \varepsilon^{i j k} f^{(+) j} \eta_{a b}^{k} \partial_{b} \phi\right. \\
& \left.+f^{(-) j} \eta_{a c}^{i} \bar{\eta}_{b c}^{j} \partial_{b} \phi\right), \\
\mathbb{F}_{5 a}^{(-) i}= & \frac{\kappa}{12} e^{\frac{5}{6} \phi}\left(6 D_{a}^{(-)} f^{(-) i}+7 f^{(-) i} \partial_{a} \phi-2 \varepsilon^{i j k} f^{(-) j} \bar{\eta}_{a b}^{k} \partial_{b} \phi\right. \\
& \left.+f^{(+) j} \bar{\eta}_{a c}^{i} \eta_{b c}^{j} \partial_{b} \phi\right),
\end{aligned}
$$

and

$$
D_{a}^{( \pm)} f^{( \pm) i}=\partial_{a} f^{( \pm) i}-2 \varepsilon^{i j k} A_{a}^{( \pm) j} f^{( \pm) k} .
$$

The expansion components $\mathbb{F}_{5 a}^{( \pm) i}$ are not completely independent due to the constraints (3.29). It is straightforward to read off the constraints using Table I in Appendix A that gives four relations from the first four rows, so totally $20=24-4$ independent components remain. The last line in Table I gives rise to the constraint (3.55). Finally, we have

\footnotetext{
${ }^{4}$ It can also be derived from Eq. (3.31) by using the algebraic properties of the 't Hooft symbols in Appendix A:

$$
\begin{aligned}
\frac{1}{4} \varepsilon_{a b c d 5} R_{a b c d}= & \frac{1}{2}\left(\mathfrak{f}_{(++)}^{i j} \eta_{a b}^{i} \eta_{a b}^{j}-\mathfrak{f}_{(+-)}^{i j} \eta_{a b}^{i} \bar{\eta}_{a b}^{j}\right. \\
& \left.+\mathfrak{f}_{(++)}^{i j} \bar{\eta}_{a b}^{i} \eta_{a b}^{j}-\mathfrak{f}_{(-)}^{i j} \bar{\eta}_{a b}^{i} \bar{\eta}_{a b}^{j}\right) \\
= & 2\left(\mathfrak{f}_{(++)}^{i j} \delta^{i j}-\mathfrak{f}_{(-)}^{i j} \delta^{i j}\right)=0 .
\end{aligned}
$$
}




$$
\begin{aligned}
R_{5 a 5 b}= & \mathfrak{R}_{6+a, 6+b} \equiv \Re_{a b} \\
= & e^{\frac{1}{3} \phi}\left\{\frac { \kappa ^ { 2 } } { 4 } e ^ { \phi } \left(\left(f^{(+) i} f^{(+) i}+f^{(-) i} f^{(-) i}\right) \delta_{a b}\right.\right. \\
& \left.+2 f^{(+) i} f^{(-) j} \eta_{a c}^{i} \bar{\eta}_{b c}^{j}\right) \\
& \left.-\frac{2}{9} \partial_{a} \phi \partial_{b} \phi+\frac{1}{18} \partial_{c} \phi \partial_{c} \phi \delta_{a b}-\frac{1}{3} D_{a} \partial_{b} \phi\right\},
\end{aligned}
$$

where $\mathfrak{R}_{a b}=\mathfrak{R}_{b a}$, so totally ten components. Thus, we recover the $50=20+20+10$ components of Riemann curvature tensors in five dimensions.

\section{FIVE-DIMENSIONAL EINSTEIN MANIFOLDS}

It was shown in Eq. (A36) that $\psi_{A B}^{\mathrm{A}}$ defined in Eq. (3.23) provide the five-dimensional representation of $\operatorname{sp}(2) \cong$ $s o(5)$ Lie algebra. It is the irrep of $s p(2)$ corresponding to the highest weight $\varpi_{2}=(0,1)$ on the right-hand side in Fig. 4. It is well known [13] that a simple Lie algebra of rank $r$ possesses $r$ inequivalent fundamental irreps. The two fundamental weights for the Lie algebra $s p(2) \cong s o(5)$ are shown in Fig. 4. The four-dimensional representation, corresponding to the highest weight $\varpi_{1}=(1,0)$ on the left-hand side in Fig. 4, is the spinor representation of $s o(5)$ and the defining representation of $s p(2)$. In contrast, $\varpi_{2}=$ $(0,1)$ is the highest weight of a five-dimensional representation of $s p(2) \cong s o(5)$ Lie algebra. It is easy to find the defining representation of $s o(5)$ that is given by Eq. (A37). There must exist a five-dimensional representation of $s p(2)$ defined by the fundamental weight $\varpi_{2}=(0,1)$. That is precisely provided by the psi symbol (3.23).

Therefore, the expansion (3.27) corresponds to the generalization of the four-dimensional decomposition (2.14) to the five-dimensional case. The five-dimensional curvature tensors are not decomposed into some irreducible blocks, because the Lorentz group $S O(5)=S p(2) / \mathbb{Z}_{2}$ is a simple group, unlike the four-dimensional case.
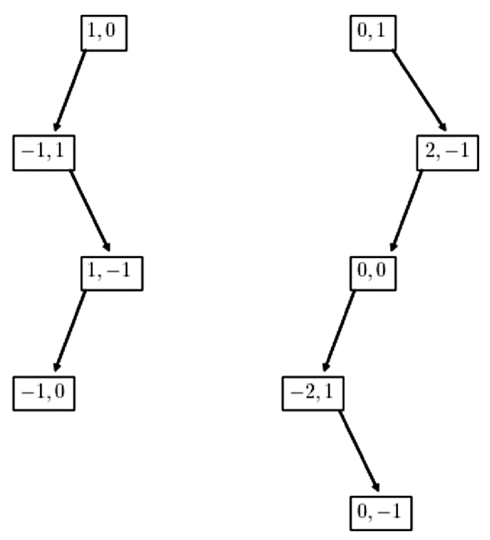

FIG. 4. The block weight diagrams of the fundamental representations of $s p(2) \cong s o(5)$.
The Riemann curvature tensor belongs to the irrep of $S O(5)=S p(2) / \mathbb{Z}_{2}$ given by

$$
R_{A B C D} \in(50=\square) .
$$

Thus, the five-dimensional Einstein manifold satisfying the equations of motion (3.5) should take elements in the irrep (3.5). The expansion (3.27) shows how these elements are organized according to the root structure in Fig. 2 or 3. After the Kaluza-Klein compactification, the symmetry is reduced to $S O(4) \times U(1)$. Then the Riemann curvature tensors in Eq. (4.1) are decomposed according to the remaining symmetry (3.10) or, more precisely, Eq. (3.44). This decomposition appears in Eqs. (3.50), (3.57), and (3.60). In particular, the components $f_{( \pm \pm)}^{i j}$ of four-dimensional Einstein manifolds appear in the curvature tensor (3.50). The instanton structure of Einstein manifolds is deformed by the excitations of $U(1)$ gauge fields and a scalar field. However, these deformations in the curvature tensor (3.50) are done only in the root directions $A_{ \pm}$and $B_{ \pm}$, corresponding to the $x$ axis and the $y$ axis, respectively, in the root diagram in Fig. 2 or 3 . Therefore, if the mixed components $\mathfrak{f}_{(+-)}^{i j}=\mathfrak{f}_{(-+)}^{j i}$ in the deformed curvature tensor $R_{a b c d}$ identically vanish, the instanton structure is still maintained despite the presence of $U(1)$ gauge fields and a scalar field, i.e.,

$$
\mathfrak{\mho}_{a b}^{( \pm) i}= \pm \frac{1}{2} \varepsilon_{a b c d} \mathfrak{\mho}_{c d}^{( \pm) i},
$$

where $\mathfrak{F}_{a b}^{(+) i} \equiv \frac{1}{4} R_{a b c d} \eta_{c d}^{i} \quad$ and $\quad \mathfrak{F}_{a b}^{(-) i} \equiv \frac{1}{4} R_{a b c d} \bar{\eta}_{c d}^{i}$. But generic excitations of four-dimensional fields break the instanton structure of four-dimensional Einstein manifolds. Consequently, once the fifth dimension is opened so that the Lorentz symmetry is enhanced to $S O(5)$, all these deformations have to be organized into a single fivedimensional Einstein manifold. So it may be interesting to look at some particular cases.

First, consider the case with $\phi=$ const. A caveat is that the condition $\phi=$ const implies the unwanted result, $\frac{1}{4} F_{\mu \nu} F^{\mu \nu}=\frac{\Lambda}{\kappa^{2}} e^{-\frac{4}{3} \phi}$, from Eq. (3.15). In order to avoid this conclusion, one can proceed in the reverse order by putting the condition $\phi=$ const in the action (3.8) or the ansatz (3.6) and varying the action afterward [12]. The constant scalar field can simply be removed by a field redefinition and defining a four-dimensional cosmological constant $\lambda \equiv \frac{3}{2} e^{-\frac{\kappa}{\sqrt{3}} \Phi} \Lambda$. If we turn off the $U(1)$ gauge field, i.e., $A_{\mu}=0$, we recover the four-dimensional Einstein manifolds discussed in Sec. II. We know well the instanton structure of four-manifolds in this case. So let us turn on the $U(1)$ gauge field. Note that the Einstein equation (3.13) can be equivalently written as

$$
R_{a b}-\frac{1}{2} \delta_{a b} R+\lambda e^{\frac{1}{3} \phi} \delta_{a b}=0 .
$$


Using the result (C3), it is straightforward to reduce Eq. (4.3) as the form $[9,10]$

$$
f_{(++)}^{i j} \delta^{i j}=f_{(--)}^{i j} \delta^{i j}=\frac{\lambda}{2}, \quad f_{(+-)}^{i j}=16 \pi G_{4} f^{(+) i} f^{(-) j} .
$$

Since the Maxwell equations are coming from the components of the Ricci tensor $R_{a 5}=R_{5 b a b}$, we can read off the expansion for the Maxwell equations in the $s p(2) \cong s o(5)$ basis from Eq. (3.57):

$$
\eta_{a b}^{i} D_{b}^{(+)} f^{(+) i}+\bar{\eta}_{a b}^{i} D_{b}^{(-)} f^{(-) i}=0,
$$

where the covariant derivatives are defined by Eq. (3.59).

The structure in Eq. (4.4) clearly shows that turning on $U(1)$ gauge fields introduces a mixing of $S U(2)_{+}$ and $S U(2)_{-}$sectors, since the mixed part $f_{(+-)}^{i j}$ no longer vanishes. Although the Riemann curvature tensor in this case does not satisfy the self-duality equation like Eq. (4.2), the mixed part $f_{(+-)}^{i j}$ does not disturb the conformal and instanton structures of four-manifolds, since the Weyl tensor does not depend on the mixed part $f_{(+-)}^{i j}=f_{(-+)}^{j i}$ [9]. A bit mysterious aspect is that there is no effect in the four-dimensional Einstein equations (4.4) if only self-dual (i.e., $f^{(-) i}=0$ ) or anti-self-dual (i.e., $\left.f^{(+) i}=0\right) U(1)$ gauge fields are turned on. This structure is due to the fact that the energy-momentum tensor in Eq. (C4) identically vanishes for self-dual or anti-self-dual gauge fields. So one may conclude that the Einstein structure is infinitely degenerate in the sense that one can add arbitrary self-dual or anti-self-dual $U(1)$ gauge fields without spoiling the Einstein condition of a four-manifold. But the five-dimensional Einstein manifold secretly notices the existence of such $U(1)$ instantons, because the four-dimensional Maxwell equations (4.5) correspond to $R_{a 5}=0$ as shown by Eq. (B19) and they are nontrivial. ${ }^{5}$

The deformed instanton structure defined by Eq. (4.2) does not allow a similar frigidity as long as $U(1)$ gauge

\footnotetext{
${ }^{5}$ This kind of absurd insensitivity holds true even when we consider a four-dimensional gravity coupled to $S U(2)$ YangMills gauge theory [9]. The Einstein equations in this case are simply replaced by $f_{(+-)}^{i j}=16 \pi G_{4} \operatorname{Tr}\left(f^{(+) i} f^{(-) j}\right)$, where the trace is performed for the $S U(2)$ gauge group. If $S U(2)$ gauge fields are Yang-Mills instantons whose equations are exactly the same as Eq. (2.22), $f_{(+-)}^{i j}$ again identically vanishes. Therefore, the Einstein structure is infinitely degenerate even for the presence of Yang-Mills instantons. It is quite strange considering that the instanton equation of Yang-Mills gauge fields is exactly the same as that of an Einstein manifold. But a higher-dimensional Einstein manifold secretly notices the existence of such Yang-Mills instantons as the five-dimensional case, because the fourdimensional Yang-Mills equations are obtained by the KaluzaKlein compactification of a higher-dimensional gravity [26].
}

fields and a scalar field are active. For simplicity, let us consider the case where only the scalar field is turned on but $U(1)$ gauge fields are completely turned off. Among the field configurations obeying the condition $\mathfrak{f}_{(+-)}^{i j}=0$ which is equal to the equations

$$
f_{(+-)}^{i j}=-\frac{1}{24}\left(D_{a} \partial_{b} \phi+\frac{1}{6} \partial_{a} \phi \partial_{b} \phi\right) \eta_{a c}^{i} \bar{\eta}_{b c}^{j}
$$

with the covariant derivative $D_{a} \partial_{b} \phi=\partial_{a} \partial_{b} \phi-\omega_{c b a} \partial_{c} \phi$, the instanton Eq. (4.2) would not be affected by the scalar field if it satisfied the equation

$$
\Delta \phi=\frac{1}{6}\left(\partial_{a} \phi\right)^{2}
$$

But Eq. (4.7) cannot be satisfied for a nontrivial physical scalar field, because the left-hand side upon integration with a proper boundary (or asymptotic) condition becomes negative while the right-hand side is positive definite. This implies that the instantons in four-dimensional Einstein manifolds are all connected by activating the fourdimensional fields in the metric (3.7). Then it will be possible to bind $S U(2)_{+}$instantons and $S U(2)_{-}$antiinstantons into a single multiplet of the five-dimensional Lorentz group $S O(5)=S p(2) / \mathbb{Z}_{2}$. The unification of two independent instantons in a four-dimensional Einstein manifold would be clear when looking at the root diagram in Fig. 2 or 3. In four dimensions, one can move only along the $x$ direction or the $y$ direction which lies in the representation of $s u(2)_{+}$or $s u(2)_{-}$, respectively, in Eq. (3.49). These two classes cannot be mixed, because the corresponding root vectors are orthogonal to each other. But, in five dimensions, one can now move along the diagonal directions which correspond to the coset elements in Eq. (3.49). Thus, it will be possible to connect two kinds of instantons by exciting four-dimensional fields coupled with $s o(5) \cong s p(2)$ root vectors. It will be left for future work to explicitly analyze the unification of four-dimensional Einstein manifolds in five dimensions.

\section{DISCUSSION}

There is a mysterious transition between Euclidean spaces and Minkowski (Lorentzian) spaces. They are simply related by an analytic continuation $x^{0}=-i x^{4}$, but it results in dramatic changes of physics. In the Euclidean space, physical forces have the self-dual structure defined by Eq. (2.4). The eigenspace of the self-dual structure is called instantons. The 2-forms are important in Riemannian geometry because of their relation with the curvature tensor, and this decomposition has a profound influence on the underlying geometry of four dimensions [6]. And this separation is deeply related to the splitting of the Euclidean Lorentz group (1.1). This correspondence is natural from the viewpoint of the Clifford isomorphism 
[20], since the 2-forms $\Omega^{2}$ in the exterior algebra are isomorphically related to the Lorentz generators $J_{a b}=$ $\frac{1}{4}\left[\gamma_{a}, \gamma_{b}\right]$ in the Clifford algebra. After the Wick rotation $x^{0}=-i x^{4}$, the physical forces no longer have the self-dual structure, because the Hodge $*$ operator satisfies $*^{2}=-1$. Instead, a novel structure emerges in the Minkowski (Lorentzian) space, the so-called causal structure. A vector or, more generally, tensors have the causal structure depending on their signature: timelike if $\|x\|^{2}<0$, spacelike if $\|x\|^{2}>0$, and lightlike if $\|x\|^{2}=0$. The causal structure in Euclidean spaces is trivial, because always $\|x\|^{2}>0$ unless $x=0$. Moreover, the Lorentz group $S O(3,1)$ becomes a simple group, although it is a noncompact group. The physical forces are no longer separated, but they exert their influences according to the causality. We wonder what the relationship between these two structures is. A five-dimensional Lorentzian manifold may provide some clue for the question, since time-independent solutions can be classified by the fourdimensional self-dual structure and $S O(4) \subset S O(4,1)$.

Our formalism can also be applied to noncompact Riemannian manifolds with a boundary. However, in this case, it is necessary to include boundary terms to discuss the topological invariants such as the Euler characteristic $\chi(M)$ and the Hirzebruch signature $\tau(M)$ [14,27-29]. These boundary terms introduce a mixing between

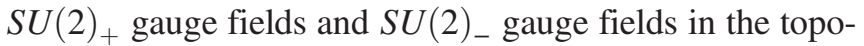
logical invariants, because the Lorentz symmetry $S O(4) \cong$ $S U(2)_{+} \otimes S U(2)_{-} / \mathbb{Z}_{2}$ is reduced to $S O(3) \cong S U(2) / \mathbb{Z}_{2}$ on the boundary [21]. The boundary symmetry $S U(2)$ corresponds to the diagonal element of $S U(2)_{+} \otimes S U(2)_{-}$. Thus, the nice splitting between $S U(2)_{+}$and $S U(2)_{-}$ factors is lost. Furthermore, all known examples, at least, for gravitational instantons, imply [27-29] that $\chi(M)=|\tau(M)|+1$. The reduction of the topological invariants is due to the reduction of the Lorentz symmetry at the boundary. The topologically inequivalent sector of instanton solutions is defined by the homotopy class of a map from a three-sphere at asymptotic infinity to the gauge group $G$ :

$$
f: \mathbb{S}^{3} \rightarrow G,
$$

and the topological charge is given by an element of the homotopy group $\pi_{3}(G)$ [18]. Since the spin connection (2.7) can be viewed as gauge fields in $G=S O(4)$, the topological sector of the $S O(4)$ gauge fields is given by the homotopy class $\pi_{3}(S O(4))=\pi_{3}\left(S U(2)_{+} \times S U(2)_{-}\right)=$ $\mathbb{Z} \oplus \mathbb{Z}$. Consequently, there are two independent topological charges, $\chi(M)$ and $\tau(M)$. But, if a noncompact Riemannian manifold has a boundary, the Lorentz symmetry $S O(4)$ is reduced to $S O(3)$ due to the boundary and the homotopy class has to be defined by the remaining symmetry, i.e., $\pi_{3}(S O(3))=\mathbb{Z}$. This implies that the Euler characteristic $\chi(M)$ and the Hirzebruch signature $\tau(M)$ are no longer independent, but there must be some relation between them. The relation $\chi(M)=|\tau(M)|+1$ represents such a relationship. It will be interesting to understand such a boundary effect from the Kaluza-Klein theory. In particular, it is an interesting problem to include boundary terms in the action (3.4) and understand a role of $U(1)$ gauge fields and a scalar field at the boundary.

The proton is a stable particle, because it cannot decay to light leptons due to the baryon number conservation. However, in the GUT, a large simple group such as $S U(5)$ or $S O(10)$ contains quarks and leptons in the same multiplet. Therefore, it is possible for the proton to decay into a lepton (a positron and two gamma-ray photons), although its half-life is extremely long. A similar instability of Einstein manifolds may appear in a five-dimensional gravity. In five dimensions, the Lorentz group is $S O(5) \supset$ $S O(4)$, which is a simple group. Therefore, $S U(2)_{+}$ instantons and $S U(2)_{-}$anti-instantons must be embedded in the same multiplet of $S O(5)$. The reason for the stability of a four-dimensional Einstein manifold is that instantons and anti-instantons belong to different gauge groups as we intentionally indicated. However, in five dimensions, they belong to an irrep of the same simple group. Then it is impossible to prevent these instantons from decaying each other. The topological consideration also supports this conjecture. In five dimensions, the Euler characteristic identically vanishes: $\chi=0$. It is a simple consequence of Poincare duality that manifolds with an odd dimension have vanishing Euler characteristic. The Hirzebruch signature can also be defined only in multiples of four dimensions. The homotopy consideration $f: \mathbb{S}^{4} \rightarrow G$ similar to Eq, (5.1) also supports this kind of triviality, because $\pi_{4}(S O(5))=\mathbb{Z}_{2}$ (see Table 4.1 in Ref. [18]). Thus, there is no natural topological invariant to support the stability of a five-dimensional Einstein manifold. If the fifth dimension is compactified with a sufficiently small radius, the Lorentz symmetry (3.10) is reduced to $S O(4) \times U(1)$. Then Einstein manifolds may recover their stability in four dimensions. But this kind of instability in five dimensions may have appeared in the early Universe, and the Universe would have stabilized in four dimensions through some similar mechanism [30]. It would be great if one could explain in this way why our Universe has chosen the fourdimensional spacetime.

The standard Kaluza-Klein vacuum $M \times \mathbb{S}^{1}$ is known to be unstable [31-34]. The instanton that mediates the decay is the five-dimensional Euclidean Schwarzschild solution

$$
d s^{2}=\frac{d r^{2}}{1-\frac{R^{2}}{r^{2}}}+r^{2} d \Omega_{3}^{2}+\left(1-\frac{R^{2}}{r^{2}}\right) d \chi^{2},
$$

where $d \Omega_{3}^{2}$ is the metric on the unit three-sphere and $\chi$ is the coordinate on the Kaluza-Klein circle. The fivedimensional solution (5.2) is a bounce which describes 
the decay of the Kaluza-Klein vacuum and is a topology changing process. In four dimensions, the Euclidean Schwarzschild solution [17] is Ricci flat, and it consists of an $S U(2)_{+}$instanton and an $S U(2)_{-}$anti-instanton. The solution is semiclassically stable, since it carries nontrivial topological invariants, $\chi(M)=1+1=2$ and $\tau(M)=0$ $[14,21]$. However, if the four-dimensional Schwarzschild solution is lifted to the five-dimensional solution (5.2), it was shown in Ref. [31] that a nonperturbative instability of the ground state is developed. Therefore, it will be interesting to investigate the nature of instability in Refs. [31-34] from the perspective we have discussed above. Any progress in this direction will be reported.

\section{ACKNOWLEDGMENTS}

We thank the anonymous referee for a careful reading of the manuscript and helpful criticisms with some explanations. H.S. Y. was supported in part by the National Research Foundation of Korea (NRF) with Grant No. NRF-2018R1D1A1B0705011314. We also appreciate APCTP for its hospitality during completion of this work.

\section{APPENDIX A: $S O(4) \cong S U(2)_{+} \otimes S U(2)_{-} / \mathbb{Z}_{2}$ AND $S O(5)=S p(2) / \mathbb{Z}_{2}$}

The defining representation of the Lie algebra $s o(n)$ is

$$
\text { so }(n)=\left\{M \mid M \in \mathfrak{g l}(n, \mathbb{R}) \text { such that } M^{T}=-M\right\} .
$$

We take six generators of the Lie algebra so(4) as

$$
\left(X_{i}\right)_{a b}=-\varepsilon_{i a b 4}, \quad\left(Y_{i}\right)_{a b}=-\left(\delta_{a i} \delta_{b 4}-\delta_{b i} \delta_{a 4}\right),
$$

where $i=1,2,3, a, b=1, \ldots, 4$, and the Levi-Civita tensor is normalized as $\varepsilon_{1234}=1$. Two sets $\left(X_{i}, Y_{i}\right)$ satisfy the commutation relations

$$
\left[X_{i}, X_{j}\right]=\varepsilon_{i j k} X_{k}, \quad\left[Y_{i}, Y_{j}\right]=\varepsilon_{i j k} X_{k}, \quad\left[X_{i}, Y_{j}\right]=\varepsilon_{i j k} Y_{k},
$$

where $\varepsilon_{i j k}=\varepsilon_{i j k 4}$. It is convenient to define a new set of generators as

$$
\tau_{i}^{ \pm}=-\frac{1}{2}\left(X_{i} \pm Y_{i}\right)
$$

Then $\tau_{i}^{ \pm}$satisfy so(3) or $s u(2)$ Lie algebra, separately:

$$
\left[\tau_{i}^{ \pm}, \tau_{j}^{ \pm}\right]=-\varepsilon_{i j k} \tau_{k}^{ \pm}, \quad\left[\tau_{i}^{ \pm}, \tau_{j}^{\mp}\right]=0 .
$$

Hence, the Lie algebra $s o(4)$ is a direct sum of two independent $s o(3)$ or $s u(2)$ Lie algebras:

$$
s o(4) \cong s o(3)_{+} \oplus s o(3)_{-} \cong s u(2)_{+} \oplus s u(2)_{-} .
$$

Since the direct sum of Lie algebras corresponds to the direct product of Lie groups and the universal covering group of $S O(3)$ is $S U(2)$, we get the group isomorphism [24]

$$
S O(4) \cong S U(2)_{+} \otimes S U(2)_{-} / \mathbb{Z}_{2} .
$$

One can identify the components of two families of $4 \times 4$ matrices $\tau_{i}^{ \pm}$from Eq. (A1):

$$
\begin{aligned}
& 2\left[\tau_{+}^{i}\right]_{a b} \equiv \eta_{a b}^{i}=\varepsilon^{i a b 4}+\left(\delta^{i a} \delta^{4 b}-\delta^{i b} \delta^{4 a}\right), \\
& 2\left[\tau_{-}^{i}\right]_{a b} \equiv \bar{\eta}_{a b}^{i}=\varepsilon^{i a b 4}-\left(\delta^{i a} \delta^{4 b}-\delta^{i b} \delta^{4 a}\right) .
\end{aligned}
$$

Explicitly, they are given by $[10,21]$

$$
\begin{aligned}
\tau_{+}^{1} & =\frac{1}{2}\left(\begin{array}{cccc}
0 & 0 & 0 & 1 \\
0 & 0 & 1 & 0 \\
0 & -1 & 0 & 0 \\
-1 & 0 & 0 & 0
\end{array}\right), \tau_{+}^{2}=\frac{1}{2}\left(\begin{array}{cccc}
0 & 0 & -1 & 0 \\
0 & 0 & 0 & 1 \\
1 & 0 & 0 & 0 \\
0 & -1 & 0 & 0
\end{array}\right), \\
\tau_{+}^{3} & =\frac{1}{2}\left(\begin{array}{cccc}
0 & 1 & 0 & 0 \\
-1 & 0 & 0 & 0 \\
0 & 0 & 0 & 1 \\
0 & 0 & -1 & 0
\end{array}\right),
\end{aligned}
$$

$\tau_{-}^{1}=\frac{1}{2}\left(\begin{array}{cccc}0 & 0 & 0 & -1 \\ 0 & 0 & 1 & 0 \\ 0 & -1 & 0 & 0 \\ 1 & 0 & 0 & 0\end{array}\right), \quad \tau_{-}^{2}=\frac{1}{2}\left(\begin{array}{cccc}0 & 0 & -1 & 0 \\ 0 & 0 & 0 & -1 \\ 1 & 0 & 0 & 0 \\ 0 & 1 & 0 & 0\end{array}\right)$,

$\tau_{-}^{3}=\frac{1}{2}\left(\begin{array}{cccc}0 & 1 & 0 & 0 \\ -1 & 0 & 0 & 0 \\ 0 & 0 & 0 & -1 \\ 0 & 0 & 1 & 0\end{array}\right)$.

The matrices in Eq. (A6) provide two independent spin $s=\frac{3}{2}$ representations of $s u(2)$ Lie algebra. The so-called 't Hooft symbols defined by Eq. (A6) satisfy the following relations [10,21]:

$$
\begin{gathered}
\eta_{a b}^{( \pm) i}= \pm \frac{1}{2} \varepsilon_{a b}{ }^{c d} \eta_{c d}^{( \pm) i} \\
\eta_{a b}^{( \pm) i} \eta_{c d}^{( \pm) i}=\delta_{a c} \delta_{b d}-\delta_{a d} \delta_{b c} \pm \varepsilon_{a b c d} \\
\varepsilon_{a b c d} \eta_{d e}^{( \pm) i}=\mp\left(\delta_{e c} \eta_{a b}^{( \pm) i}+\delta_{e a} \eta_{b c}^{( \pm) i}-\delta_{e b} \eta_{a c}^{( \pm) i}\right) \\
\eta_{a b}^{( \pm) i} \eta_{a b}^{(\mp) j}=0 \\
\eta_{a c}^{( \pm) i} \eta_{b c}^{( \pm) j}=\delta^{i j} \delta_{a b}+\varepsilon^{i j k} \eta_{a b}^{( \pm) k} \\
\eta_{a c}^{( \pm) i} \eta_{b c}^{(\mp) j}=\eta_{b c}^{( \pm) i} \eta_{a c}^{(\mp) j}
\end{gathered}
$$


$\varepsilon^{i j k} \eta_{a b}^{( \pm) j} \eta_{c d}^{( \pm) k}=\delta_{a c} \eta_{b d}^{( \pm) i}-\delta_{a d} \eta_{b c}^{( \pm) i}-\delta_{b c} \eta_{a d}^{( \pm) i}+\delta_{b d} \eta_{a c}^{( \pm) i}$,

(A15)

where $\eta_{a b}^{(+) i} \equiv \eta_{a b}^{i}$ and $\eta_{a b}^{(-) i} \equiv \bar{\eta}_{a b}^{i}$.

Now we consider the so(5) Lie algebra. The fivedimensional gamma matrices $\gamma_{A}=\left(\gamma_{a}, \gamma_{5}\right), A=1, \ldots, 5$, are given by

$\gamma_{a}=\left(\begin{array}{cc}0 & \sigma^{a} \\ \bar{\sigma}^{a} & 0\end{array}\right), \quad \gamma_{5}=-\gamma_{1} \gamma_{2} \gamma_{3} \gamma_{4}=\left(\begin{array}{cc}\mathbf{1}_{2} & 0 \\ 0 & -\mathbf{1}_{2}\end{array}\right)$,

where $\sigma^{a}=\left(i \tau^{i}, \mathbf{1}_{2}\right)$ and $\bar{\sigma}^{a}=\left(-i \tau^{i}, \mathbf{1}_{2}\right)=\left(\sigma^{a}\right)^{\dagger}$ with $\tau^{i}$ the Pauli matrices. They satisfy the Dirac algebra

$$
\left\{\gamma_{A}, \gamma_{B}\right\}=2 \delta_{A B}
$$

Then the Lorentz generators of so(5) Lie algebra are defined by

$$
J_{A B}=\frac{1}{4}\left[\gamma_{A}, \gamma_{B}\right]
$$

One can see that the four-dimensional Lorentz algebra generated by $J_{a b}$ obeys the chiral representation [see Eq. (3.19)] whose generators are given by

$$
J_{a b}^{( \pm)}=\frac{1}{2}\left(1 \pm \gamma_{5}\right) J_{a b}
$$

and

$J_{a b}^{(+)}=\frac{i}{2} \eta_{a b}^{i} \tau^{i} \in s u(2)_{+}, \quad J_{a b}^{(-)}=\frac{i}{2} \bar{\eta}_{a b}^{i} \tau^{i} \in s u(2)_{-}$.

The 't Hooft symbols in Eq. (A6) are obtained by

$$
\eta_{a b}^{i}=-i \operatorname{Tr}\left(J_{a b}^{(+)} \tau^{i}\right), \quad \bar{\eta}_{a b}^{i}=-i \operatorname{Tr}\left(J_{a b}^{(-)} \tau^{i}\right) .
$$

The chiral generators in Eq. (A19) independently satisfy the four-dimensional Lorentz algebra that verifies the Lie algebra isomorphism (A4).

It is easy to check the Lie algebra isomorphism $s p(2) \cong$ so(5) using the identification (3.20). The matrices $T^{\mathbb{A}}$ in Eq. (3.21) are anti-Hermitian, i.e., $\left(T^{\mathbb{A}}\right)^{\dagger}=-T^{\mathbb{A}}$, and obey the relation

$$
\left(T^{\mathbb{A}}\right)^{T} J+J T^{\mathbb{A}}=0,
$$

where

$$
J=i\left(\tau^{2} \otimes \mathbf{1}_{2}\right)=i\left(\begin{array}{cc}
\tau^{2} & 0 \\
0 & \tau^{2}
\end{array}\right)
$$

is the symplectic matrix. The relation (A22) implies that the $4 \times 4$ matrices $T^{\AA}$ in Eq. (3.21) are the Lie algebra generators of $s p(2)$. Indeed, they satisfy the $s p(2)$ Lie algebra

$$
\left[T^{\mathbb{A}}, T^{\mathbb{B}}\right]=-f^{\mathbb{A B C}} T^{\mathbb{C}},
$$

where $f^{\mathrm{ABC}}$ are totally antisymmetric structure constants. Their nonzero components are listed below:

$f^{i j k}=f^{(3+i)(3+j) k}=f^{(6+i)(6+j) k}=\varepsilon^{i j k}, \quad f^{(3+i)(6+j) 10}=\delta^{i j}$

that can be read off from Eq. (3.32). Thus, we establish the Lie algebra isomorphism $s p(2) \cong s o(5)$.

The $s o(5)$ generators in Eq. (A18) also obey the anticommutation relation

$\left\{J_{A B}, J_{C D}\right\}=-\frac{1}{2}\left(\delta_{A C} \delta_{B D}-\delta_{A D} \delta_{B C}\right) \mathbf{1}_{4}-\frac{1}{2} \varepsilon_{A B C D E} \gamma_{E}$.

The corresponding anticommutation relations for the $s p(2)$ generators in Eq. (3.21) read as

$$
\left\{T^{\mathbb{A}}, T^{\mathbb{B}}\right\}=-\frac{1}{2} \delta^{\mathbb{A B}} \mathbf{1}_{4}-\frac{1}{2} d^{\mathbb{A B C} C} \tilde{T}^{C},
$$

where $\tilde{T}^{C}$ do not belong to the set of $s p(2)$ generators. Indeed, they are given by $\tilde{T}^{A}=\gamma_{A}$ and correspond to the second term on the right-hand side in Eq. (A25). The nonvanishing components of $d^{\mathbb{A B C}}$ are listed in Table I. Then one can deduce the product of $s o(5)$ and $s p(2)$ generators:

$$
\begin{aligned}
J_{A B} J_{C D}= & -\frac{1}{4}\left(\delta_{A C} \delta_{B D}-\delta_{A D} \delta_{B C}\right) \mathbf{1}_{4} \\
& -\frac{1}{2}\left(\delta_{A C} J_{B D}-\delta_{A D} J_{B C}-\delta_{B C} J_{A D}+\delta_{B D} J_{A C}\right) \\
& -\frac{1}{4} \varepsilon_{A B C D E} \gamma_{E},
\end{aligned}
$$

TABLE I. $\pm(\mathbb{A}, \mathbb{B}, C) \equiv \pm d^{\mathbb{A B C} C}=1$.

\begin{tabular}{lrr}
\hline \hline$-(1,10,1)$ & $(5,9,1)$ & $-(6,8,1)$ \\
$-(2,10,2)$ & $-(4,9,2)$ & $(6,7,2)$ \\
$-(3,10,3)$ & $(4,8,3)$ & $-(5,7,3)$ \\
$(1,7,4)$ & $(2,8,4)$ & $(3,9,4)$ \\
$(1,4,5)$ & $(2,5,5)$ & $(3,6,5)$ \\
\hline \hline
\end{tabular}




$$
T^{\mathbb{A}} T^{\mathbb{B}}=-\frac{1}{4} \delta^{\mathrm{AB}} \mathbf{1}_{4}-\frac{1}{2} f^{\mathrm{ABC}} T^{\mathbb{C}}-\frac{1}{4} d^{\mathbb{A B C}} \tilde{T}^{C} .
$$

Using the linear relation (3.22), let us write the products in Eqs. (A27) and (A28) as the form

$$
\begin{gathered}
\text { (I) } J_{A B} J_{C D}=\psi_{A B}^{\mathbb{A}} \psi_{C D}^{\mathbb{B}} T^{\mathbb{A}} T^{\mathbb{B}}, \\
\text { (II) } T^{\mathbb{A}} T^{\mathbb{B}}=\frac{1}{4} \psi_{A B}^{\mathbb{A}} \psi_{C D}^{\mathbb{B}} J_{A B} J_{C D} .
\end{gathered}
$$

Applying the relations in Eqs. (A27) and (A28) on both sides in products (I) and (II) leads to useful algebraic relations for the psi symbols (3.23). From product (I), we get

$$
\begin{gathered}
\psi_{A B}^{A} \psi_{C D}^{\AA}=\delta_{A C} \delta_{B D}-\delta_{A D} \delta_{B C}, \\
f^{\mathrm{ABC}} \psi_{A B}^{\mathbb{B}} \psi_{C D}^{\mathbb{C}}=\delta_{A C} \psi_{B D}^{\mathrm{A}}-\delta_{A D} \psi_{B C}^{\mathrm{A}}-\delta_{B C} \psi_{A D}^{\mathrm{A}}+\delta_{B D} \psi_{A C}^{\mathrm{A}},
\end{gathered}
$$

where $\operatorname{Tr}\left(\gamma_{E} T^{\mathbb{A}}\right)=\operatorname{Tr}\left(\tilde{T}^{C} T^{\mathbb{A}}\right)=0$ were used. Similarly, from product (II), we get

$$
\begin{gathered}
\psi_{A B}^{A} \psi_{A B}^{\mathbb{B}}=2 \delta^{A \mathbb{B}}, \\
f^{A B B C} \psi_{A B}^{\mathbb{C}}=\psi_{A C}^{A} \psi_{B C}^{\mathbb{B}}-\psi_{B C}^{A} \psi_{A C}^{\mathbb{B}},
\end{gathered}
$$

where $\operatorname{Tr}\left(\gamma_{E} J_{A B}\right)=\operatorname{Tr}\left(\tilde{T}^{C} J_{A B}\right)=0$ were used. The above relations are analogous to those in Eqs. (A9)-(A15). Actually, those identities have been derived by applying a similar technique to $\mathrm{Eq}$. (A20). If we define $5 \times 5$ matrices by

$$
\left(T^{\AA}\right)_{A B} \equiv \psi_{A B}^{\AA},
$$

Eq. (A34) reduces to the commutation relations

$$
\left[T^{\mathbb{A}}, T^{\mathbb{B}}\right]=-f^{\mathbb{A B C}} T^{\mathbb{C}},
$$

while Eq. (A33) gives us the trace $\operatorname{Tr}\left(T^{\mathbb{A}} T^{\mathbb{B}}\right)=-2 \delta^{\mathbb{A B}}$. Therefore, the generators in Eq. (A35) provide the fivedimensional representation of $s p(2)$ Lie algebra which is isomorphic to the defining representation of $s o(5)$ Lie algebra with generators given by

$$
\left(J_{A B}\right)_{C D}=\delta_{A C} \delta_{B D}-\delta_{A D} \delta_{B C} .
$$

The relation (A31) corresponds to the Fierz identity for the $s p(2)$ Lie algebra generators in Eq. (A35) and the identity (A32) can be transformed into Eq. (A34) by using the trace (A33) or vice versa by using the Fierz identity (A31).

\section{APPENDIX B: KALUZA-KLEIN GRAVITY}

On a five-dimensional Riemannian manifold $N$, the spin connection $\Omega=\frac{1}{2} \Omega_{A B} J^{A B}=\frac{1}{2} \Omega_{A B M} J^{A B} d X^{M}$ constitutes an $S O(5)$ gauge field with respect to the local $S O(5)$ rotations

$$
\Omega \rightarrow \Omega^{\prime}=\Lambda \Omega \Lambda^{-1}+\Lambda d \Lambda^{-1},
$$

where $\Lambda=\exp \left(\frac{1}{2} \lambda_{A B}(X) J^{A B}\right) \in S O(5)$ and $J^{A B}$ are $\operatorname{so}(5)$ Lorentz generators in Eq. (A18). Then the covariant derivatives for the vectors $E_{A}$ and $E^{A}$ are defined by

$$
\begin{aligned}
D_{M} \mathcal{E}_{A} & =\partial_{M} \mathcal{E}_{A}-\Omega^{B}{ }_{A M} \mathcal{E}_{B}, \\
D_{M} E^{A} & =\partial_{M} E^{A}+\Omega^{A}{ }_{B M} E^{B} .
\end{aligned}
$$

The connection 1-forms $\Omega^{A}{ }_{B}=\Omega^{A}{ }_{B M} d X^{M}$ satisfy the Cartan structure equations $[14,18]$

$$
\begin{gathered}
T^{A}=d E^{A}+\Omega^{A}{ }_{B} \wedge E^{B}, \\
R_{B}^{A}=d \Omega_{B}^{A}+\Omega^{A}{ }_{C} \wedge \Omega^{C}{ }_{B},
\end{gathered}
$$

where $T^{A}$ are the torsion 2-forms and $R_{B}^{A}$ are the curvature 2-forms. We impose the torsion-free condition $T_{M N}^{A}=$ $D_{M} E_{N}^{A}-D_{N} E_{M}^{A}=0$ to recover the standard content of general relativity which determines $\Omega_{M}$ as

$$
\Omega_{A B C}=\Omega_{A B M} \mathcal{E}_{C}^{M}=\frac{1}{2}\left(f_{B C A}+f_{C A B}-f_{A B C}\right),
$$

where $f_{A B C}$ are the structure functions defined by

$$
\left[\mathcal{E}_{A}, \mathcal{E}_{B}\right]=-f_{A B}{ }^{C} \mathcal{E}_{C}
$$

or its dual equations

$$
d E^{A}=\frac{1}{2} f_{B C}{ }^{A} E^{B} \wedge E^{C} .
$$

In order to formulate gravity as a gauge theory of local Lorentz symmetry, it is necessary to introduce a local basis for the Kaluza-Klein geometry (3.6):

$$
E^{A}=\left(E^{a}, E^{5}\right)=\left(e^{-\frac{1}{6} \phi} e^{a}, e^{\frac{1}{3} \phi} e^{5}\right),
$$

where

$$
d s_{4}^{2}=e^{a} \otimes e^{a}=g_{\mu \nu}(x) d x^{\mu} d x^{\nu}
$$

and

$$
e^{5}=d x^{5}+\kappa A_{\mu}(x) d x^{\mu} .
$$

By solving the torsion-free condition $T^{A}=0$ in Eq. (B1), one can determine the spin connections as 


$$
\begin{gathered}
\Omega_{a b}=\omega_{a b}-\frac{1}{6} e^{\frac{1}{6} \phi}\left(\partial_{b} \phi E^{a}-\partial_{a} \phi E^{b}\right)-\frac{\kappa}{2} e^{\frac{2}{3} \phi} F_{a b} E^{5}, \\
\Omega_{a 5}=-\frac{1}{3} e^{\frac{1}{6} \phi} \partial_{a} \phi E^{5}-\frac{\kappa}{2} e^{\frac{2}{3} \phi} F_{a b} E^{b},
\end{gathered}
$$

where $\omega_{a b}$ is the four-dimensional spin connection for the local frames in Eq. (B7) and $\partial_{a} \equiv \mathfrak{e}_{a}=\mathfrak{e}_{a}^{\mu}(x) \partial_{\mu} \in \Gamma(T M)$ are orthonormal tangent vectors dual to the covectors $e^{a}$, i.e., $\left\langle e^{a}, \mathfrak{e}_{b}\right\rangle=\delta_{b}^{a}$. In particular, the exterior derivative acting on $M \times \mathbb{S}^{1}$ is defined by $d=d x^{\mu} \partial_{\mu}=e^{a} \partial_{a}$, since we have assumed the cylinder condition (i.e., no dependence on $x^{5}$ ).

After a little algebra, the curvature 2-forms for the Kaluza-Klein geometry (3.6) can be determined by the structure equation (B2):

$$
\begin{aligned}
R_{a b}= & { }^{(4)} R_{a b}-\frac{\kappa^{2}}{4} e^{\phi}\left(F_{a b} F_{c d}+F_{a c} F_{b d}\right) e^{c} \wedge e^{d}-\frac{\kappa}{2} e^{\phi} D_{c} F_{a b} e^{c} \wedge e^{5} \\
& -\frac{\kappa}{4} e^{\phi}\left(2 F_{a b} \partial_{c} \phi-F_{b c} \partial_{a} \phi+F_{a c} \partial_{b} \phi\right) e^{c} \wedge e^{5} \\
& +\frac{\kappa}{12} e^{\phi}\left(F_{a c} \partial_{c} \phi e^{b} \wedge e^{5}-F_{b c} \partial_{c} \phi e^{a} \wedge e^{5}\right) \\
& +\frac{1}{36}\left(\partial_{b} \phi \partial_{c} \phi e^{a} \wedge e^{c}-\partial_{a} \phi \partial_{c} \phi e^{b} \wedge e^{c}-\partial_{c} \phi \partial_{c} \phi e^{a} \wedge e^{b}\right) \\
& +\frac{1}{6}\left(D_{c} \partial_{a} \phi e^{c} \wedge e^{b}-D_{c} \partial_{b} \phi e^{c} \wedge e^{a}\right), \\
R_{5 a}= & \frac{\kappa^{2}}{4} e^{\frac{3}{2} \phi} F_{a b} F_{b c} e^{c} \wedge e^{5}+\frac{\kappa}{2} e^{\frac{1}{2} \phi} D_{c} F_{a b} e^{c} \wedge e^{b} \\
& +\frac{\kappa}{4} e^{\frac{1}{2} \phi}\left(F_{b c} \partial_{a} \phi-F_{a b} \partial_{c} \phi\right) e^{b} \wedge e^{c}-\frac{\kappa}{12} e^{\frac{1}{2} \phi} F_{b c} \partial_{b} \phi e^{a} \wedge e^{c} \\
& +\frac{1}{9} e^{\frac{1}{2} \phi}\left(2 \partial_{a} \phi \partial_{b} \phi+3 D_{b} \partial_{a} \phi\right) e^{b} \wedge e^{5}-\frac{1}{18} e^{\frac{1}{2} \phi} \partial_{b} \phi \partial_{b} \phi e^{a} \wedge e^{5} .
\end{aligned}
$$

Here, ${ }^{(4)} R_{a b}$ is the curvature 2-form determined by the four-dimensional metric (B7), and the covariant derivatives are defined by

$$
\begin{aligned}
D_{a} \partial_{b} \phi & =\partial_{a} \partial_{b} \phi-\omega_{c b a} \partial_{c} \phi, \\
D_{c} F_{a b} & =\partial_{c} F_{a b}-\omega_{d a c} F_{d b}-\omega_{d b c} F_{a d} .
\end{aligned}
$$

Note that the derivations $\partial_{a} \equiv \mathfrak{e}_{a}$ do not commute, but they satisfy the structure equation similar to Eq. (B4),

$$
\left[\partial_{a}, \partial_{b}\right]=-\mathfrak{f}_{a b}^{c} \partial_{c} .
$$

The curvature 2-forms above have the following expansion in the basis $\left(e^{c} \wedge e^{d}, e^{c} \wedge e^{5}\right)$ :

$$
\begin{aligned}
& R_{a b}=\frac{1}{2} e^{-\frac{1}{3} \phi} R_{a b c d} e^{c} \wedge e^{d}+e^{\frac{1}{6} \phi} R_{a b c 5} e^{c} \wedge e^{5}, \\
& R_{5 a}=\frac{1}{2} e^{-\frac{1}{3} \phi} R_{5 a b c} e^{b} \wedge e^{c}+e^{\frac{1}{6} \phi} R_{5 a 5 b} e^{5} \wedge e^{b} .
\end{aligned}
$$

Therefore, one can read off the components of Riemann curvature tensors from Eqs. (B11) and (B12):

$$
\begin{aligned}
R_{a b c d}= & e^{\frac{1}{3} \phi}\left\{{ }^{(4)} R_{a b c d}-\frac{\kappa^{2}}{4} e^{\phi}\left(2 F_{a b} F_{c d}+F_{a c} F_{b d}-F_{a d} F_{b c}\right)\right. \\
& +\frac{1}{36}\left(\partial_{a} \phi \partial_{c} \phi \delta_{b d}-\partial_{a} \phi \partial_{d} \phi \delta_{b c}-\partial_{b} \phi \partial_{c} \phi \delta_{a d}+\partial_{b} \phi \partial_{d} \phi \delta_{a c}-\partial_{e} \phi \partial_{e} \phi\left(\delta_{a c} \delta_{b d}-\delta_{a d} \delta_{b c}\right)\right) \\
& \left.+\frac{1}{6}\left(D_{c} \partial_{a} \phi \delta_{b d}-D_{d} \partial_{a} \phi \delta_{b c}-D_{c} \partial_{b} \phi \delta_{a d}+D_{d} \partial_{b} \phi \delta_{a c}\right)\right\},
\end{aligned}
$$




$$
\begin{gathered}
R_{a b c 5}=-\frac{1}{2} \kappa e^{\frac{5}{6} \phi}\left\{F_{a b} \partial_{c} \phi-\frac{1}{2} F_{b c} \partial_{a} \phi+\frac{1}{2} F_{a c} \partial_{b} \phi-\frac{1}{6}\left(F_{a d} \delta_{b c}-F_{b d} \delta_{a c}\right) \partial_{d} \phi+D_{c} F_{a b}\right\}, \\
R_{5 a 5 b}=e^{\frac{1}{3} \phi}\left\{\frac{\kappa^{2}}{4} e^{\phi} F_{a c} F_{b c}-\frac{2}{9} \partial_{a} \phi \partial_{b} \phi+\frac{1}{18} \partial_{c} \phi \partial_{c} \phi \delta_{a b}-\frac{1}{3} D_{b} \partial_{a} \phi\right\} .
\end{gathered}
$$

Note that

$D_{a} \partial_{b} \phi-D_{b} \partial_{a} \phi=\left(-\mathfrak{f}_{a b c}+\omega_{c a b}-\omega_{c b a}\right) \partial_{c} \phi=0$,

because $\mathfrak{f}_{a b c}=\omega_{c a b}-\omega_{c b a}$. Therefore, $R_{5 a 5 b}=R_{5 b 5 a}$ as it should be.

Now it is easy to determine the Ricci tensors and the Ricci scalar using the above results:

$$
\begin{gathered}
R_{a b}=R_{c a c b}+R_{5 a 5 b} \\
=e^{\frac{1}{3} \phi}\left({ }^{(4)} R_{a b}-\frac{\kappa^{2}}{2} e^{\phi} F_{a c} F_{b c}-\frac{1}{6} \partial_{a} \phi \partial_{b} \phi+\frac{1}{6} D_{c} \partial_{c} \phi \delta_{a b}\right), \\
R_{a 5}=R_{b a b 5} \\
=\frac{\kappa}{2} e^{\frac{5}{6} \phi}\left(F_{a b} \partial_{b} \phi+D_{b} F_{a b}\right), \\
R_{55}=R_{a 5 a 5} \\
=e^{\frac{1}{3} \phi}\left(\frac{\kappa^{2}}{4} e^{\phi} F_{a b} F_{a b}-\frac{1}{3} D_{a} \partial_{a} \phi\right)
\end{gathered}
$$

$$
\begin{aligned}
R & =R_{a b} \delta^{a b}+R_{55} \\
& =e^{\frac{1}{3} \phi}\left({ }^{(4)} R-\frac{\kappa^{2}}{4} e^{\phi} F_{a b} F_{a b}-\frac{1}{6} \partial_{a} \phi \partial_{a} \phi+\frac{1}{3} D_{a} \partial_{a} \phi\right),
\end{aligned}
$$

where ${ }^{(4)} R_{a b}={ }^{(4)} R_{c a c b}$ and ${ }^{(4)} R={ }^{(4)} R_{a a}$ are the Ricci tensors and the Ricci scalar, respectively, determined by the four-dimensional geometry (B7) and the Laplacian operator is defined by

$$
\Delta \phi \equiv D_{a} \partial_{a} \phi=\frac{1}{\sqrt{g}} \partial_{\mu}\left(\sqrt{g} g^{\mu \nu} \partial_{\nu} \phi\right)
$$

\section{APPENDIX C: REPRESENTATION OF RICCI TENSORS}

The Riemann curvature tensors in Eq. (3.24), under the group $S O(5)$, correspond to the tensor product

$$
R_{A B C D} \in \mathbf{1 0} \otimes \mathbf{1 0}=\square \otimes \square .
$$

The Clebsch-Gordan decomposition of this tensor product is given by

$$
R_{A B C D} \in \mathbf{1 0} \otimes \mathbf{1 0}=\boxminus \otimes \boxminus=(\mathbf{5 0}=\boxminus) \oplus(\mathbf{4 5}=\boxminus) \oplus(\mathbf{5}=\boxminus)
$$

The last two representations, $\mathbf{4 5} \oplus \mathbf{5}$, are removed by the first Bianchi identity (3.26). In particular, the last one, 5, corresponds to the five constraints (3.29). The Clebsch-Gordan decomposition (C2) can be further decomposed according to the symmetry breaking pattern (3.10) or, more precisely, Eq. (3.44), as was shown in Sec. III. It is straightforward to determine the decomposition of the Ricci tensors and Ricci scalar in Eqs. (B18) and (B21) using the result (3.50):

$$
\begin{aligned}
R_{a b}= & e^{\frac{1}{3} \phi}\left\{\left(f_{(++)}^{i j} \delta^{i j}+f_{(--)}^{i j} \delta^{i j}\right) \delta_{a b}+2 f_{(+-)}^{i j} \eta_{a c}^{i} \bar{\eta}_{b c}^{j}-\frac{1}{6}\left(\partial_{a} \phi \partial_{b} \phi-\Delta \phi \delta_{a b}\right)\right. \\
& \left.-\frac{\kappa^{2}}{2} e^{\phi}\left(\left(f^{(+) i} f^{(+) i}+f^{(-) i} f^{(-) i}\right) \delta_{a b}+2 f^{(+) i} f^{(-) j} \eta_{a c}^{i} \bar{\eta}_{b c}^{j}\right)\right\}, \\
R= & e^{\frac{1}{3} \phi}\left\{4\left(f_{(++)}^{i j} \delta^{i j}+f_{(--)}^{i j} \delta^{i j}\right)-\frac{1}{6}\left(\partial_{a} \phi \partial_{a} \phi-2 \Delta \phi\right)-\kappa^{2} e^{\phi}\left(f^{(+) i} f^{(+) i}+f^{(-) i} f^{(-) i}\right)\right\} .
\end{aligned}
$$

The energy-momentum tensor in Eq. (3.16) takes the form [10]

$$
\begin{aligned}
T_{a b} & =e^{\sqrt{3} \kappa \Phi} F_{a c} F_{b c}+\partial_{a} \Phi \partial_{b} \Phi-\delta_{a b}\left(\frac{1}{4} e^{\sqrt{3} \kappa \Phi} F_{c d} F_{c d}+\frac{1}{2}\left(\partial_{c} \Phi\right)^{2}\right) \\
& =2 e^{\sqrt{3} \kappa \Phi} f^{(+) i} f^{(-) j} \eta_{a c}^{i} \bar{\eta}_{b c}^{j}+\partial_{a} \Phi \partial_{b} \Phi-\frac{1}{2} \delta_{a b}\left(\partial_{c} \Phi\right)^{2} .
\end{aligned}
$$


[1] M. Gell-Mann, Symmetries of baryons and mesons, Phys. Rev. 125, 1067 (1962).

[2] R. E. Behrends, J. Dreitlein, C. Fronsdal, and B. W. Lee, Simple groups and strong interaction symmetries, Rev. Mod. Phys. 34, 584 (1962).

[3] M. Gell-Mann, A schematic model of baryons and mesons, Phys. Lett. 8, 214 (1964).

[4] M. Y. Han and Y. Nambu, Three-triplet model with double SU(3) symmetry, Phys. Rev. 139, B1006 (1965).

[5] H. Fritzsch, M. Gell-Mann, and H. Leutwyler, Advantages of the color octet gluon picture, Phys. Lett. 47B, 365 (1973).

[6] M. F. Atiyah, N. Hitchin, and I. M. Singer, Self-duality in four-dimensional Riemannian geometry, Proc. R. Soc. A 362, 425 (1978).

[7] A. L. Besse, Einstein Manifolds (Springer-Verlag, Berlin, 1987).

[8] S. K. Donaldson and P. B. Kronheimer, The Geometry of Four-Manifolds (Oxford University Press, Oxford, 1990).

[9] J. J. Oh and H. S. Yang, Einstein manifolds as Yang-Mills instantons, Mod. Phys. Lett. A 28, 1350097 (2013).

[10] J. Lee, J. J. Oh, and H. S. Yang, An efficient representation of Euclidean gravity I, J. High Energy Phys. 12 (2011) 025.

[11] H. S. Yang, Riemannian manifolds and gauge theory, Proc. Sci., CORFU2011 (2011) 063.

[12] Modern Kaluza-Klein Theories, edited by T. Appelquist, A. Chodos, and P. G. O. Freund (Addison-Wesley, Reading, MA, 1987).

[13] H. Georgi, Lie Algebras in Particle Physics: From Isospin to Unified Theories, 2nd ed. (Advanced Book Program, Boulder, Colorado, 1999).

[14] T. Eguchi, P. B. Gilkey, and A. J. Hanson, Gravitation, gauge theories and differential geometry, Phys. Rep. 66, 213 (1980).

[15] L. Andersson and V. Moncrief, Einstein spaces as attractors for the Einstein flow, J. Diff. Geom. 89, 1 (2011).

[16] K. Kröncke, On the stability of Einstein manifolds, Ann. Glob. Anal. Geom. 47, 81 (2015).

[17] S. W. Hawking, Gravitational instantons, Phys. Lett. 60A, 81 (1977).

[18] M. Nakahara, Geometry, Topology and Physics (IOP Publishing, Bristol, 1990).
[19] R. Utiyama, Invariant theoretical interpretation of interaction, Phys. Rev. 101, 1597 (1956).

[20] E. Meinrenken, Clifford Algebra and Lie Theory (Springer, Berlin, 2013).

[21] J. J. Oh, C. Park, and H. S. Yang, Yang-Mills instantons from gravitational instantons, J. High Energy Phys. 04 (2011) 087.

[22] N. Hitchin, Compact four-dimensional Einstein manifolds, J. Diff. Geom. 9, 435 (1974).

[23] D. Page, A compact rotating gravitational instanton, Phys. Lett. 79B, 235 (1979).

[24] B. G. Wybourne, Classical Groups for Physicists (John Wiley \& Son, New York, 1974).

[25] E. Witten, Search for a realistic Kaluza-Klein theory, Nucl. Phys. B186, 412 (1981).

[26] Y. M. Cho and P. G. O. Freund, Non-Abelian gauge fields as Nambu-Goldstone fields, Phys. Rev. D 12, 1711 (1975).

[27] G. W. Gibbons and C. N. Pope, Positive action conjecture and asymptotically Euclidean metrics in quantum gravity, Commun. Math. Phys. 66, 267 (1979).

[28] G. W. Gibbons and S. W. Hawking, Classification of gravitational instanton symmetries, Commun. Math. Phys. 66, 291 (1979).

[29] G. W. Gibbons, C. N. Pope, and H. Römer, Index theorem boundary terms for gravitational instantons, Nucl. Phys. B157, 377 (1979).

[30] K. K. Kim, S. Koh, and H. S. Yang, Expanding universe and dynamical compactification using Yang-Mills instantons, J. High Energy Phys. 12 (2018) 085.

[31] E. Witten, Instability of the Kaluza-Klein vacuum, Nucl. Phys. B195, 481 (1982).

[32] F. Dowker, J.P. Gauntlett, G. W. Gibbons, and G. T. Horowitz, Decay of magnetic fields in Kaluza-Klein theory, Phys. Rev. D 52, 6929 (1995).

[33] M. Dine, P. J. Fox, and E. Gorbatov, Catastrophic decays of compactified space-times, J. High Energy Phys. 09 (2004) 037.

[34] I. G. Etxebarria, M. Montero, K. Sousa, and I. Valenzuela, Nothing is certain in string compactifications, J. High Energy Phys. 12 (2020) 032. 